To Appear in The Astrophysical Journal

\title{
Population Synthesis in the Blue II. The Spectroscopic Age of 47 Tucanae
}

\author{
Ricardo P. Schiavon, S. M. Faber \\ UCO/Lick Observatory, University of California, Santa Cruz, CA 95064. \\ ripisc,faber@ucolick.org \\ James A. Rose \\ Department of Physics and Astronomy, CB 3255, University of North Carolina, Chapel \\ Hill, NC 27599 \\ jim@physics.unc.edu \\ and \\ Bruno V. Castilho \\ Laboratório Nacional de Astrofísica, MCT CP 21, 37500-000 Itajubá, Brazil \\ bruno@lna.br
}

\begin{abstract}
We develop a new set of models for intermediate-metallicity single stellar populations in the blue/optical region and use those models to determine the spectroscopic age of 47 Tuc. The models are based on a moderately high-resolution (1.8 $\AA$ FWHM) empirical spectral library, state-of-the-art theoretical isochrones from M. Salaris and the most recent set from the Padova group, and new semiempirical calibrations between fundamental stellar parameters and observables. Model line-strengths include all corrections for deficiencies of the stellar library that are described in Paper I. We highlight the importance of correctly modeling the luminosity function (LF) of the cluster at the level of the giant branch, in order to achieve a good reproduction of the integrated spectrum; agreement between the spectroscopic age and the age based on the cluster's color-magnitude diagram (CMD) is achieved only if the observed LF is used rather than the theoretical ones, which either do not include AGB stars (Salaris) or underpredict the
\end{abstract}


total number counts of bright giants in the cluster by a factor of two (Padova). After all corrections are made, the CMD and the spectroscopic ages (from $\mathrm{H} \gamma$ and $H \beta$ ) are in close agreement: $\sim 11-12$ Gyrs for Salaris isochrones and $\sim 13$ Gyrs for Padova. The difference between the model ages is due to the inclusion of atomic diffusion in the Salaris models. Previously older spectroscopic ages were due to the underestimate of the number of red giants and/or the use of isochrones that neglected the effects of He-diffusion and $\alpha$-enhancement. Uncertainties in spectroscopic age determinations of old stellar populations stem from a number of effects, the most important of which are the $T_{\text {eff }}$ and $[\mathrm{Fe} / \mathrm{H}]$-scales of the giant stars used in the stellar library, the LF on the upper giant branch, and the assumed metallicity of the target stellar population itself. A \pm 1 Gyr uncertainty in age results from uncertainties of $\pm 75 \mathrm{~K}$ in the $T_{\text {eff }}$-scale of the library giants, \pm 0.1 dex in the level of the giant-branch $\mathrm{LF}$, and \pm 0.1 dex in the assumed $[\mathrm{Fe} / \mathrm{H}]$ of either the target stellar population or the assumed zeropoint of the metallicity scale of the stellar library. A similar underestimate in the bright giant LF, if it exists in current super-solar metallicity models, would cause spectroscopic ages of elliptical galaxies inferred from such models to be too high by approximately $30 \%$.

Subject headings: clusters: globular

\section{Introduction}

In Paper I of this series (Schiavon et al. 2002), we performed an empirical synthesis of the integrated spectrum of the Galactic metal-rich globular cluster 47 Tucanae. The model spectrum was computed directly from a color-magnitude diagram (CMD) of the cluster,

being thus essentially independent of inputs from theoretical isochrones. In that paper we achieved good agreement between models and observations in the equivalent widths of two Balmer lines and several metal lines. In order to reach such agreement, we needed to correct the model line indices for two limitations of the spectral library adopted in our models, namely, the relative paucity of sufficiently metal-poor giants and the absence of CN-strong stars, which are known to be present in the cluster. We also had to require that the iron abundance of 47 Tuc be $[\mathrm{Fe} / \mathrm{H}]=-0.75$, which is slightly below and yet within the errorbars of the standard value determined by Carretta \& Gratton $(1997)([\mathrm{Fe} / \mathrm{H}]=-0.7)$.

In this second paper of the series, we turn to the more challenging task of making models based on theoretical isochrones. All the corrections described above, which were inferred in Paper I, are applied to the models computed here. Moreover, we subtract from the 
observed indices the contribution by blue stragglers, which are not included in the theoretical isochrones. It was shown in Paper I that such corrections are important only for $H \delta_{F}$ and, to a lesser degree, $H \gamma_{\sigma<130}$. The effect of blue stragglers on all indices studied in this paper is shown in Table 1. The index values adopted in this paper are those listed in the second row of Table 3 of Paper I.

The resultant model spectra computed from the theoretical isochrones are used to deduce the cluster's age. Such a test is of fundamental importance for stellar population models. A key requirement is that the same age has to be obtained from the fit of the theoretical isochrone to the cluster's CMD, and from the fit of theoretical spectra to the integrated spectrum of the cluster (the so-called spectroscopic age). This is especially interesting in view of the exceedingly high spectroscopic age ( $>20$ Gyrs) that was obtained for 47 Tuc from the equivalent width of $H \gamma$ by Gibson et al. (1999). The mismatch between spectroscopic and CMD-based ages may even be more general, because Cohen, Blakeslee \& Rhyzov (1998) have found very high spectroscopic ages for a sample of Galactic GCs, on the basis of the equivalent width of $H \beta$. More recently, Vazdekis et al. (2001) showed that a lower spectroscopic age $(\sim 13$ Gyrs $)$ is obtained when isochrones that take into consideration effects such as $\alpha$-enhancement and diffusion of heavy elements are used. However, Vazdekis et al. still obtained a residual difference of $\sim 4$ Gyrs between their spectroscopic and CMDbased ages, in the sense that the spectroscopic age was higher.

In the present paper, we approach this problem as follows. We first infer an age for the cluster by fitting theoretical isochrones to its CMD. We adopt the isochrones by Salaris and collaborators, which are the same as those employed by Vazdekis et al. (2001). For comparison purposes, we perform the same exercise adopting $\alpha$-enhanced isochrones by Salasnich et al. (2000, hereafter, Padova isochrones). The isochrones are compared to cluster data in both the CMD and luminosity function (LF) domains. A major conclusion is that the raw isochrones adopted do not reproduce the cluster's LF correctly. They underestimate the number counts of giants brighter than the horizontal branch by roughly a factor of 2.5 , in part because the theoretical isochrones do not include AGB stars. When we adopt the Padova isochrones, which do include AGB stars, the mismatch is reduced by $\sim 0.1$ dex. We then infer corrections to the model predictions by forcing the theoretical LF to match the observed one. Such corrections are shown to be very important, implying a decrease of the order of 3-4 Gyrs in the spectroscopic age inferred from Balmer lines. The LF of giant stars is thus a key input in deriving accurate spectroscopic ages of old stellar populations. The revised spectroscopic ages inferred when adopting the "LF-corrected" isochrones are in excellent agreement with those obtained from the fits to the CMD. Via this careful two-step procedure in Papers I and II, we now feel that the mysterious spectroscopic age discrepancy for 47 Tuc has been resolved. 
The paper is organized as follows. In Section 2 the theoretical isochrones are compared to data on 47 Tuc in both the CMD and LF domains. In Section 3 we compare observed line-indices to model predictions computed from both the original and the "LF-corrected" isochrones. Model uncertainties due to errors in the input stellar parameters are discussed in Section 4, and our conclusions are summarized in Section 5.

\section{Theoretical Isochrones vs. Data}

\subsection{Color-Magnitude Diagram}

Before comparing the isochrone-based model spectra with the observations, we call attention to important features of the theoretical isochrones that are crucial to interpreting our results. In Figure 1 the CMD of 47 Tuc (Howell, Guhathakurta \& Gilliland 2000) is overlaid with isochrones from Salaris and collaborators for 8, 10, 12 and 14 Gyrs. Conversion from the theoretical plane $\left(L_{b o l}, T_{e f f}\right)$ to the observational plane $[V,(B-V)]$ was performed accord-

ing to the recipe described in Paper I, and adopting a distance modulus of $(m-M)_{0}=13.33$ and $E(B-V)=0.04$ (Kaluzny et al. 1998). These isochrones are described in Vazdekis et al. (2001) and were kindly provided by M. Salaris. They are computed taking into consideration diffusion of heavy elements and an $\alpha$-enhanced mixture $([\mathrm{Fe} / \mathrm{H}]=-0.7,[\alpha / \mathrm{Fe}]=+0.4)$. These were the isochrones used by Vazdekis et al. (2001) to infer a spectroscopic age of $\sim 14$ Gyrs for 47 Tuc, on the basis of the measurement of the $H \gamma_{\sigma<130}$ index (for a definition of that index, see Figure 16 of Paper I). The age inferred from their fit to the CMD of the cluster is significantly younger: $\sim 10$ Gyrs. In spite of this discrepancy, Vazdekis et al. (2001) greatly improved over previous spectroscopic age determinations, which gave ages in excess of 20 Gyrs (Gibson et al. 1999). In particular, they showed that $\alpha$-enhancement and diffusion of heavy elements cause a reduction in both the CMD and spectroscopic ages of 47 Tuc by several Gyrs.

From Figure 1 it can be seen that the best match to the position of the cluster's turn-off in the CMD is achieved for an isochrone with age 11-12 Gyrs. This is of course dependent on the reddening assumed for the cluster. An error of 0.01 mag in $E(B-V)$ results in an uncertainty of $\sim 1$ Gyr in the age inferred from CMD-fitting. That is the reason why we infer a slightly older age than Vazdekis et al., even though we are using the same set of isochrones, as their adopted $E(B-V)$ is $0.01 \mathrm{mag}$ higher than ours. The isochrone for 11 Gyrs matches very well the color of the turn-off of the cluster. However, it predicts a subgiant branch which is too bright by $0.05 \mathrm{mag}$. There also are mismatches in the color of the giant branch. The model isochrone is too red by $\sim 0.03$ mag for giants brighter than $\mathrm{V} \sim 14$ and too blue by a comparable amount at the base of the giant branch. Adopting the Alonso et 
al. (1999) calibration of $T_{e f f} \mathrm{~s}$ vs. $(B-V)$, rather than the recipe of Paper I, leads to a slightly better match in the base of the giant branch, but it worsens the match at $V \sim 14$, where our calibration provides a good match to the data. Such mismatches, if due to errors in the $T_{\text {eff }} \mathrm{s}$ of the isochrones, may have a non-negligible impact on the ages inferred from the fit of the integrated spectrum of the cluster, as we show below. It is also important to notice that AGB stars are not included in the Salaris isochrones. It will be shown in Section 3 that this also has an important impact on the integrated line indices.

In Figure 2 we repeat the CMD of 47 Tuc, this time comparing the data with the Padova isochrones. Theoretical quantities $\left(T_{\text {eff }}\right.$ and $\left.M_{b o l}\right)$ were transformed into the observational plane following the same recipes as adopted to the Salaris isochrones shown in Figure 1. The Padova isochrones were computed assuming the same chemical composition as adopted in the computation of the Salaris isochrones. However, the Padova isochrones do not consider the effect of diffusion of helium and heavier elements. As explained by Vazdekis et al. (2001), inclusion of heavy-element diffusion results in a slightly cooler and fainter turn-off for a given stellar mass and chemical composition. In fact, the turn-off of the 11 Gyrs isochrone from Salaris is about 0.16 mag fainter and $150 \mathrm{~K}$ cooler than the turn-off of the Padova isochrone for a similar age (11.2 Gyrs). For this reason the age that best fits the turn-off color and magnitude of 47 Tuc, according to the Padova isochrones, is somewhere between 12.5 and 14.1 Gyrs, being thus slightly older than the one inferred from the Salaris isochrones (Figure 1).

Evidence for the occurence of atomic diffusion in the Sun has been presented in a number of studies. For instance, Basu, Pinsonneault \& Bahcall (2000) show that diffusion of heavy elements is required if models are to fit the data on the profiles of sound speed, density and adiabatic index in the Sun. For more metal-poor stars, Lebreton et al. (1999) showed that the HR diagram from Hipparcos data for stars with $-1.05<[\mathrm{Fe} / \mathrm{H}]<-0.45$ can be reconciled with the predictions of stellar evolution only if diffusion of heavy elements is considered. However, the extent to which atomic diffusion affects stellar structure and evolution is still under debate. It has been argued that the constancy of the Li abundance in metal-poor dwarfs with a wide range of metallicities (the so-called lithium plateau, Spite \& Spite 1982) suggests that diffusion effects may be partially inhibited (Deliyannis \& Demarque 1991). On the other hand, Salaris \& Weiss (2001) showed that models with fully efficient diffusion may be consistent with the lithium plateau if the errors on lithium abundances and sample incompleteness are duly accounted for. According to Vazdekis et al. (2001), the consideration of atomic diffusion in its full extent reduces the turn-off age of 47 Tuc by $\sim$ 1 Gyr. An important constraint on the extent of diffusion affects has also been placed by Gratton et al. (2001), who found that $[\mathrm{Fe} / \mathrm{H}]$ is the same for stars at the turn-off and at the base of the giant branch of the metal-poor globular cluster NGC 6397. Models by Chaboyer 
et al. (2001) show that the latter result implies an inhibition of diffusion in the outer layers of stellar models, such that the reduction of CMD-based ages due to atomic diffusion is only $4 \%$ ( $\sim 0.5 \mathrm{Gyr}$ in the case of $47 \mathrm{Tuc}$ ). In summary, though we tend to favor the age based on Salaris models (11 Gyrs), the main emphasis of this work is on making sure that the spectroscopic age we obtain is consistent with the one based on the fit of the cluster turn-off, for whatever set of isochrones is employed.

In Figure 2 it also can be seen that the Padova isochrones do not perfectly fit the color of the giant branch, the mismatch being similar (if slightly worse) to the one mentioned above for the Salaris isochrones: Padova isochrones are bluer than the observations by $\sim 0.04 \mathrm{mag}$ in the giant branch. We note that the Padova isochrones do include the AGB phase, but it is also displaced to the blue, as compared to the observations, by the same amount as the giant branch. The position of the HB is well matched by the Padova isochrones, but its extension is underpredicted.

\subsection{Luminosity Function}

Matching a CMD such as the one shown in Figure 1 is just the first step when comparing a given theoretical isochrone with cluster data; matching the LF of the cluster is also extremely important. In fact, the LF of giant stars has a large and not often appreciated impact on the integrated spectrum in general, and on line indices in particular. In Figure 3, we compare the LF measured from the data with the LF of the first-ascent giant-branch predicted by the best-fitting Salaris theoretical isochrone of age 11 Gyrs (thick line). The observed LF is in good agreement with data on 47 Tuc from other sources in the literature, namely Hesser et al. (1987), Kaluzny et al. (1998), and Zoccali et al. (2000) (the last being based roughly on the same data as this work). The theoretical LF is a good match to the observed one up to the horizontal branch. For brighter stars, however, the Salaris LF underpredicts the observations by $\sim 0.4$ dex on average (the mismatch is slightly lower at $V \sim 12.5$ and higher at the location of the AGB bump, at $V \sim 13$ ).

The LF mismatch is in part due to the fact that the Salaris LF does not include AGB stars. However, on purely theoretical grounds it is hard to attribute the mismatch solely to the lack of these stars, as current stellar evolution models predict a ratio of $\sim 0.15$ between the lifetimes in the AGB and HB phases (Cassisi et al. 2001). At the AGB bump (V 13.1), the observed number of AGB stars is roughly the same as first-ascent giants: in the interval $12.8<V<13.2$, there are $30 \mathrm{AGB}$ and 32 first-ascent RGB stars. Thus, adding AGB stars would imply roughly a factor of two (0.3 dex) increase, in the raw theoretical LF at the AGB-bump level. However, the difference between the observed and theoretical LFs 
at the level of the AGB bump amounts to $\sim 0.5 \operatorname{dex}(V \sim 13.1$ in Figure 3), which leaves an extra 0.2 dex mismatch between the pure RGB observed LF and the theoretical one. For brighter stars, it becomes increasingly difficult to distinguish between AGB and first-ascent RGB stars, but it is doubtful that the ratio changes very much. At fainter magnitudes, below the AGB bump, there are even fewer observed AGB stars. Therefore we conclude that only at most $2 / 3$ of the mismatch between the Salaris LF and the data at the level of the AGB bump could be due to the lack of AGB stars in the theoretical isochrone, and that fraction is probably lower in other regions of the AGB, where AGB stars are even more sparsely distributed. In short, some increase in the model prediction for the number of model first-ascent giants above the HB seems also to be needed.

This conclusion can be checked by comparing to the predicted LF of the Padova isochrones, which include both first-ascent and AGB stars explicitly. This comparison is shown in Figure 4 which illustrates theoretical LFs based on the Padova isochrones for 11.2 Gyrs. The thick solid line represents the total Padova LF, while the thick dash-dotted line represents the same LF after removal of the AGB stars. For reference, we also reproduce the original Salaris LF from Figure 3, now as a thin line. We note that because the Padova isochrones are provided in coarser mass steps, the signature of the Horizontal Branch in the Padova LF is broader than in the case of the Salaris LF, but the total number of HB stars is similar. Two conclusions can be drawn from this figure. First, we call attention to the very good agreement between the two sets of pure RGB theoretical isochrones (thin line and thick dashed line), which means that both groups predict similar lifetimes for first-ascent giant stars. This has also been shown by Zoccali \& Piotto (2000). Second, and more importantly, even when the AGB stars in the Padova isochrones are considered (thick solid line), the number counts above the Horizontal Branch are still underpredicted by $\sim 0.2-0.3$ dex. This confirms our suspicion above that the theoretical number of first-ascent giants above the HB is also too smal. Evidently the shortfall is similar in both the Salaris and Padova models.

We recall that our observed LF is in good agreement with that of other works, like Hesser et al. (1987), Kaluzny et al. (1998) and Zoccali et al. (2000). In particular, the LFs of Hesser et al. and Kaluzny et al. do not refer to the central parts of the cluster, so that it is very unlikely that the LF mismatch is due to an environmental effect, or that it may be afflicted by observational errors due to crowding.

Another feature that can affect the observed number ratio of giant to main sequence stars is binarity. Throughout this discussion, we have adopted the turn-off region $(V \sim 17.5)$ as the normalization point to compare the theoretical LF to the observations. However, if the number counts at the level of the turn-off and upper main sequence are underestimated due to the presence of binary stars, this could be responsible for part of the LF mismatch 
of Figures 3 and 4 . The color distribution of stars within narrow magnitude ranges of the main sequence of 47 Tuc indeed are not symmetrical, presenting an extended tail towards redder $(B-V)$, which is a well-known signature of binary contamination. In fact, Albrow et al. (2001) have determined a binary fraction of $14 \% \pm 4 \%$ from observations of upper main sequence stars in the core of 47 Tuc. In a study of binarity effects on the LF of NGC 6752, Rubenstein \& Bailyn (1999) found that the turn-off luminosity function needs to be corrected upwards by $\sim 0.1$ dex, if the binary fraction is $22 \%$ at about 1 mag below the turn-off. We therefore conclude that, since the binary fraction of 47 Tuc is lower than that of NGC 6752, at a comparable position in the main sequence, the correction on the LF of 47 Tuc is lower than 0.1 dex, and thus is not able to account for the mismatch seen in Figures 3 and 4. Moreover, we note that a correction for the effect of binaries, while bringing a slight improvement to the match in the upper giant branch, would bring the LF into disagreement in the lower giant branch.

The situation regarding the match of stellar evolution models to the LFs of first-ascent giants in clusters is controversial. That the theoretical models do not provide a perfect match to the number counts of giants in some clusters has been pointed out some time ago (Stetson 1991, Bergbusch \& Vandenberg 1992, Bolte 1994, Sandquist et al. 1996, Sandquist et al. 1999). In a recent paper, Langer, Bolte \& Sandquist (2000) found a comparable mismatch (i.e. $\sim 0.2$ dex underestimate above the HB) between observed and theoretical LFs of firstascent RGB stars for the metal-poor clusters M5 and M30. In particular, they note that the models underpredict the number of stars at different levels of the giant branch for the two clusters. For M30, the mismatch happens for giants below the RGB bump, whereas for M5 the models underpredict the number of giants brighter than the RGB bump, similarly to what we found for 47 Tuc. Langer et al. suggest that the mismatch can be due to deep mixing, which would bring fresh fuel to the hydrogen-burning shell, thus prolonging the lifetimes of first-ascent RGB stars. On the other hand, Zoccali \& Piotto (2000) compared the observed LFs of a number of clusters over a broad range of metallicities to theoretical models by different groups, including Padova, Straniero et al. (1997), previous models by Salaris and collaborators (Salaris \& Weiss 1998). Contrary to the conclusions of Langer et al. (2000), they found rather good agreement for all clusters more metal-poor than $[\mathrm{Fe} / \mathrm{H}]$ $\sim-0.7$. However, in their Figure 5 a slight mismatch (up to $\lesssim 0.2 \mathrm{dex}$ ), in the same sense as found in this work, can be seen in the LF of 47 Tuc for $V \lesssim 13.5$.

While there still is controversy concerning the ability of theoretical models to predict the LF of first-ascent giants, the AGB LF is largely unexplored. To our knowledge, there is as yet no direct detailed comparison in the literature between number counts of AGB stars in clusters and model LFs. This is partly due to the paucity of statistically representative samples of cluster AGB stars in the literature, mostly on account of their short lifetimes. On the 
theoretical side, AGB models are subject to important uncertainties that may affect lifetime predictions (Cassisi et al. 2001), mostly related to the unknown efficiency of macroscopic mechanisms, like convection and diffusion, and to the input physics of the models (equation of state, opacities and nuclear cross sections). On the calibration side, the matter is further complicated by the uncertainties in the bolometric corrections of $\mathrm{M}$ giants, especially for the most metal-rich clusters, where molecular opacities in the $\mathrm{V}$ band, and in particular their dependence on metallicity, are difficult to model (see, for instance, Westera et al. 2002).

Maraston (1998) compared model predictions, based on the fuel consumption theorem, to the fractional contribution of RGB and AGB stars from NGC 6528 and NGC 6553 to the integrated light in the $\mathrm{V}$ band, finding good agreement. This result is difficult to reconcile with our findings, although Maraston's models are based on a different set of theoretical models. Moreover, Maraston's test does not disentangle the effect of the number of AGBs and their V-band luminosities, which are both subject to significant uncertainties. Taken at face value, Maraston's result suggests that there may not be a LF mismatch for clusters of near-solar metallicity, although this is at odds with the finding, by Zoccali \& Piotto (2000), that model LFs are a poor match to the number counts of near-solar metallicity clusters in the upper giant branch.

Regardless of the origin of the LF discrepancy in Figure 3, its presence must be considered in order to account correctly for all evolutionary stages in the CMD of Figure 1. We also want to compare spectroscopic ages based on both the raw and corrected LFs, in order to compare our results to those of Vazdekis et al. (2001), who used the Salaris isochrones and (apparently) the associated raw LF. This will be discussed in Section 3.

\subsection{Effective Temperatures and Colors of the Red Giants}

Another important issue concerns the interplay between the $T_{\text {eff }}$-scale of the model isochrones and the $T_{\text {eff }} v s$. $(B-V)$ relation used in the conversion of the theoretical isochrones to the observational plane. As noted above, the Salaris isochrone that best matches the cluster's turn-off ( $\sim 11$ Gyrs $)$ presents a mismatch of as much as $\sim 0.03$ mag in the color of the red giant branch. However, even a successful fit to the giant branch $(B-V)$ color in the CMD of a cluster would not mean that both the theoretical $T_{\text {eff-scale and the }} T_{\text {eff }} v s$. $(B-V)$ are individually correct. As stressed by Weiss \& Salaris (1999) and Salaris, Cassisi

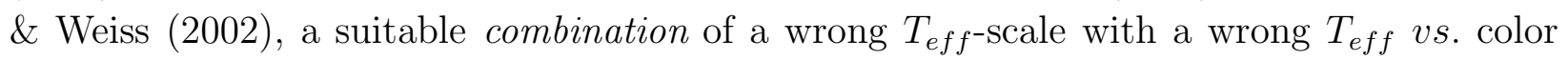
relation can produce a successful match to the giant branch. For the same reason, the slight mismatch of $0.03 \mathrm{mag}$ between the theoretical isochrone for 11 Gyrs and the data on the giant branch of 47 Tuc could be due to a wrong $T_{e f f}$-scale in the isochrone, to systematic 
errors in the $T_{\text {eff }} v s$. $(B-V)$ calibration, or to a combination of the two. To quantify the importance of this effect, let us suppose that the assumed $T_{\text {eff }} v s$. $(B-V)$ relation is correct and that the mismatch is due solely to a systematic error in the theoretical $T_{e f f} \mathrm{~s}$ of the giant stars, as given by the isochrones. In this case, we can estimate by how much the $T_{\text {eff }} \mathrm{s}$ of the giants would need to be increased (decreased) in order to shift the upper (lower) giant branch by 0.03 mag to the blue in Figure 1 . With the aid of the $T_{\text {eff }}$ vs. $(B-V)$ calibration derived in Paper I (Figure 4), we estimate that the $T_{\text {eff }} \mathrm{s}$ of the giants need to be increased (decreased) by $\sim 40 \mathrm{~K}$ in order to match the upper (lower) giant branch.

In the case of the Padova isochrones, the mismatch is different, the theoretical isochrone being systematically $\sim 0.03-0.05$ mag bluer than the observations all along the giant branch. Thus, under the same assumption as above, $T_{\text {eff }} \mathrm{s}$ predicted by the isochrones would need to be systematically decreased by $40-80 \mathrm{~K}$ in order to match the observed giant branch.

We note that such mismatches are not implausible in view of discrepancies among

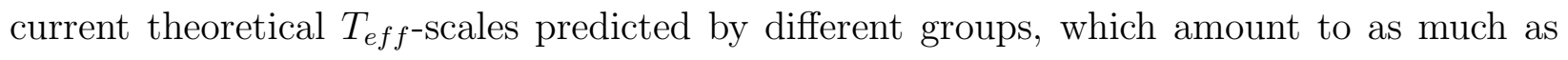
200 K (see Figure 2 of Schiavon, Barbuy \& Bruzual 2000, and discussion in Salaris et al. 2002). The consequence of such a shift for our model predictions is discussed in Section 4.

\section{Isochrone-based Model Spectra vs. Observations: The Spectroscopic Age of 47 Tucanae}

This section compares model line indices based on theoretical isochrones to the observations. Here we briefly summarize the most important ingredients of our models, referring the reader to Paper I for details. Our models are based on a moderately-high resolution (1.8 $\AA$ FWHM) stellar spectral library (Jones 1999) and two sets of isochrones: those by Salaris and collaborators, and the ones by Salasnich et al. (2000), which are computed adopting the same chemical mixture as the former but do not consider diffusion of heavy elements. The observed integrated spectra of 47 Tuc are described in Paper I.

\subsection{Models from the Original Isochrones}

The procedure to compute integrated spectra from an observed CMD was described in Paper I. The procedure used here is the same except that the number of stars per isochrone point is given by theory plus a Salpeter IMF, while in the computations from the CMD it is given by the observed number of stars. Another difference refers to the $T_{\text {eff }} \mathrm{s}$, which are provided directly by the isochrones, so that they do not rely on a $(B-V)$ vs. $T_{\text {eff }}$ relation. 
However, we do need to resort to such a relation when computing the weighted, fluxed spectrum for every isochrone point.

Our first goal in computing isochrone-based models is to recalculate the integrated model indices of Vazdekis et al. (2001) using the same isochrones and spectral library, but adopting the new set of relations derived in Paper I between fundamental stellar parameters $\left(T_{\text {eff }}\right.$ and $\left.L_{b o l}\right)$ and various observables (colors, absolute magnitudes, spectra, and line indices). Vazdekis et al. adopt the calibrations of Alonso et al. (1995, 1996, 1999) for all evolutionary stages. We showed in Paper I that our calibrations are consistent with Alonso et al.'s for dwarfs but are roughly $70 \mathrm{~K}$ warmer at fixed $(B-V)$ for giants. Another difference is the stellar parameters of the library stars, which were determined through different procedures. We will first concentrate on the computations based on the Salaris isochrones, then contrast these results with the computations from the Padova isochrones.

The model spectra are smoothed and rebinned to the resolution and dispersion of the observed spectra of 47 Tuc, and the line indices are measured using the definitions given in Paper I. Corrections are applied as in Paper I, namely, corrections due to the paucity of metal-poor giants in the library, the slightly lower metallicity of 47 Tuc (from $[\mathrm{Fe} / \mathrm{H}]=$ -0.7 to -0.75$)$, and the effect of CN-strong stars. The isochrones adopted are computed for $[\mathrm{Fe} / \mathrm{H}]=-0.7$ and $[\alpha / \mathrm{Fe}]=+0.4$. However, in Paper I we inferred corrections to bring line index predictions to $[\mathrm{Fe} / \mathrm{H}]=-0.75$, which is the effective $\mathrm{Fe}$ abundance corresponding to our model predictions. We also correct the observed indices to eliminate the contribution by blue stragglers, which of course are not included in the isochrones. The corrected EWs are given in Table 4 of Paper I. Percentage corrections for blue stragglers in each index are given in Table 1.

The first comparison is to Salaris models without AGB stars. Figures 5 and 6 show a comparison between these models versus observed indices in a number of index $v s$. index plots. According to these figures, different Balmer lines imply different ages. $H \beta$ and $H \gamma_{\sigma<130}$ are best fitted for an age of 14 Gyrs, while $H \delta_{F}$ requires an even older age. Most importantly, best-fitting spectroscopic ages are always older (by at least $\sim 3$ Gyrs) than that obtained from the fit to the color and absolute magnitude of the cluster turn-off (11-12 Gyrs). The models also provide good fits to all metal lines, although the age for which the best fit is achieved again varies from one metal line to the next. Note that the model prediction for $(B-V)$ is also too blue compared to the observations: for an age of 11 Gyrs, the model predicts $(B-V)=0.79$, which is 0.07 mag bluer than the observed value (see Table 3 of Paper I). This again implies that an older age is needed to reconcile with the observed color. On balance, these spectroscopic ages are consistent with the ones obtained by Vazdekis et al. (2001) on the basis of the same set of isochrones and spectral library but using a different 
set of stellar parameters and calibrations.

Figures 7 and 8 reproduce the data of Figures 5 and 6 but now using model predictions based on the Padova isochrones. In order to bring all isochrones to the same footing, we first compare results based on Padova isochrones after removing their AGB components. The basic conclusion from Figures 7 and 8 is that spectroscopic ages inferred from Padova isochrones are larger than those based on Salaris isochrones by a little more than 2 Gyrs. Such a difference is significantly higher than expected based on fits to the position of the cluster turn-off (Figures 1 and 2), which yielded an age difference (due to heavy element diffusion) of $\sim 1$ Gyr. The small discrepancy is plausibly explained by the systematically too warm giant branch in the Padova models, which forces an older overall spectroscopic age. The temperature difference at the level of the Horizontal Branch is of the order of $50 \mathrm{~K}$ and increases up to the tip of the Giant Branch, where it reaches $\sim 100 \mathrm{~K}$. This difference in the temperature of the red giant branch is also responsible for another feature worthy of notice in Figures 12 and 13: that the metal lines are all weaker when Padova isochrones are used (compare with Figures 10 and 11).

\subsection{Models with "Corrected" Luminosity Functions}

As discussed in Section 2, both sets of isochrones employed in this work underestimate the number of giant stars brighter than the horizontal branch. In the case of the Salaris isochrones, which do not take into account AGB stars, the mismatch is of the order of 0.4 dex, while in the case of the Padova isochrones, which do include AGBs, it is slightly lower, of the order of $0.2-0.3 \mathrm{dex}$. To evaluate the impact of these underestimates on our computations, we apply empyrical corrections to both theoretical LFs to bring them into agreement with the observations.

\subsection{Salaris Isochrones and the Spectroscopic Age of 47 Tuc}

We first correct the Salaris isochrones in order to bring their predicted LF into agreement with the observations; discussion of the Padova isochrones is postponed to the next subsection. The corrected Salaris LF for a model with 11 Gyrs is represented in Figure 3 as the thin line. The consequence of correcting our model predictions for this effect is illustrated as the arrow in Figure 9 for the $\langle F e>-H \beta$ diagram. Since the giant light contribution is increased, the integrated spectrum becomes redder, metal lines become stronger, and hydrogen lines become weaker. With this correction to the LF, the spectroscopic age based 
on $H \beta$ is reduced from $\gtrsim 14$ to $11 \mathrm{Gyr}$, in excellent agreement with the isochrone age in Figure 1.

We checked the size of the corrections by recomputing the integrated spectrum directly from the CMD as in Paper I, but excluding AGB/RGB stars so as to force agreement with the theoretical uncorrected luminosity function. Differences between the line indices obtained in Paper I and the ones computed with the exclusion of AGB/RGB stars are essentially the same as the ones obtained adopting the two LFs shown in Figure 3, which validates the corrections estimated here.

Figures 10 and 11 compare the LF-corrected model predictions with the observations for the other indices. Virtually all line indices are well fit for ages ranging from 9 to 14 Gyrs. The best fitting age for $H \gamma_{\sigma<130}$ is 11-12 Gyrs, in agreement with the age inferred from $H \beta$ and CMD-fitting. The notable exception is $H \delta_{F}$, for which the best-fitting age is slightly higher: $\sim 14$ Gyrs. The latter result was anticipated in Paper I, because the model spectrum computed directly from the CMD of the cluster also overestimates $H \delta_{F}$, and when we considered the correction of $H \delta_{F}$ due to the effect of CN-strong stars, the mismatch was further increased. However, as discussed in Section 5.3 of Paper I, we have reason to believe that our correction for this effect on $H \delta_{F}$ may be in error. In any case it was already clear in Paper I that $H \delta_{F}$ was aberrant. All the metal-line indices are well fit by models with ages of 11-12 Gyrs, including Ca4227, which was greatly improved after correction for the effect of CN-line contamination, derived in Paper I. Finally, the integrated $(B-V)$ of the cluster is also matched for an age of 11 Gyrs.

The ratio of the contribution to the integrated light of turn-off stars to giant stars (HB stars excluded) provides a good gauge of the impact of the underestimate of the number of bright stars in the theoretical isochrone. In Paper I we computed the fractional contribution to the integrated light by different evolutionary stages at a number of reference wavelengths. Those numbers are given in Table 5 of Paper I. They are fairly robust, since they are based on a statistically representative CMD of the cluster. From Table 5 of Paper I, it can be seen that the ratio of turn-off light to giant light is 0.50 and 0.28 in the regions of $H \delta_{F}$ and $H \beta$ respectively. If the uncorrected theoretical LF is adopted, those figures change to 0.77 and 0.47 respectively.

We have just shown that a 0.4 dex underestimate in the contribution of red giants to the integrated light leads to an age overestimate of about 3-4 Gyrs for fixed hydrogen line equivalent width. It is interesting to note that this age difference is the same as the one found by Vazdekis et al. (2001) between their spectroscopic and CMD-based age determinations.

The fact that the amount of red giant light contribution has such an important effect on 
spectroscopic age estimates may be surprising, given that it is often said in the literature that spectral features in the integrated light of old stellar populations evolve with age mainly as a function of the temperature of the turn-off stars. Though this statement is strictly correct, it neglects the basic fact that a significant fraction, if not most, of the continuum light of old stellar populations even in the blue is provided by giant stars (see the discussion in Section 5.5 of Paper I). Thus, our results show that the luminosity function is a key constraint on population synthesis models, upon which our ability to correctly predict the ages of old stellar populations from their integrated light is strongly dependent.

In summary, we conclude that the too-large spectroscopic age of 47 Tuc found in previous works is due to a combination of reasons: the neglect of important effects like $\alpha$-enhancement and He-diffusion (as previously shown by Vazdekis et al. 2001), and the underestimate of the contribution of bright red giants to the integrated light of the cluster.

\subsection{Padova Isochrones}

In this section we compare the observed absorption line EWs with model predictions based on Padova isochrones, now including AGB stars. As in the previous section, the LF above the Horizontal Branch has been empyrically corrected in order to reproduce the observed number counts. The comparisons are displayed in Figures 12 and 13, where it can be seen that the spectroscopic age inferred when the LF-corrected Padova isochrones are adopted is about 14 Gyrs, for both $H \beta$ and $H \gamma\left(H \delta_{F}\right.$ again being older). Based on Section 3.1 , we expect that the Padova isochrones will yield a spectroscopic age that is roughly 1-2 Gyrs older than the one that is inferred when using the Salaris isochrones.

Aside from these systematically older ages, it is important to emphasize that, for $H \beta$ and $H \gamma$ we achieve generally good consistency between spectroscopic and CMD-based ages for the Padova models, just as we did with the Salaris models. As for the model predictions for $H \delta_{F}$ they are again much higher than the observations, thus leading to too old ages according to this index.

We also performed computations using the original Padova isochrones, i.e., including AGB stars with their theoretically predicted LF (solid line in Figure 4), which underpredicts the observed LF of bright giants by $0.2-0.3$ dex. The spectroscopic age inferred in this

case is $\sim 2-3$ Gyrs older than the one inferred above, and thus is in disagreement with the CMD-based age. Therefore, we again stress the importance of correctly matching the giant branch luminosity function in order to predict spectroscopic ages which agree with the ones based on the fit of the cluster CMD. As discussed in previous sections, it is hard to quantify 
to which extent the LF mismatch is due to errors in the models predictions of AGB and first-ascent RGB stars. While AGB stars may have larger uncertainties, we have reasons to believe that the predictions for first-ascent giants may be also in error, as noted in Section 2.2 .

\section{Uncertainties and Systematic Effects due to Input Stellar Parameters}

In this section we gauge the effects of uncertainties in the $T_{\text {eff }}$ and $[\mathrm{Fe} / \mathrm{H}]$-scales adopted as model inputs. As discussed in Paper I, the $T_{\text {eff }}$ and $[\mathrm{Fe} / \mathrm{H}]$-scales of metal-poor stars from different sources in the literature may differ by up to $\sim 100 \mathrm{~K}$ and 0.1 dex. We also argued in Section 2 that the $T_{\text {eff }} \mathrm{s}$ predicted by theoretical isochrones may be in error by comparable amounts. Our set of stellar parameters was determined from Strömgren photometry for the dwarfs and from calibrations of line-indices $v s$. stellar parameters for the giants. Our $T_{\text {eff }}$ and metallicity-scales for the library giants are, by construction, tied to the stellar parameter calibrations of Soubiran et al. (1998) (see Paper I for details).

As in Paper I, we resort to fitting functions in order to assess the changes in line indices that result from slight changes in model inputs. The stellar parameters tested were the $T_{\text {eff }}$ and $[\mathrm{Fe} / \mathrm{H}]$-scales of dwarfs and giants separately, plus the assumed $[\mathrm{Fe} / \mathrm{H}]$ of 47 Tuc. For these computations, giants are considered to be all stars with $T_{\text {eff }}<6000 \mathrm{~K}$ and $\log g<$ 3.6 , and dwarfs are in the same $T_{\text {eff }}$ interval but with $\log g>3.6$ (thus including subgiants). From the discussion of systematic effects on these stellar parameters carried out in Paper I, we chose to vary the $T_{\text {eff }} \mathrm{S}$ of giants and dwarfs by \pm 75 and $\pm 50 \mathrm{~K}$ respectively. The $[\mathrm{Fe} / \mathrm{H}] \mathrm{s}$ of giants and dwarfs were varied by \pm 0.1 and \pm 0.05 dex respectively, while the $[\mathrm{Fe} / \mathrm{H}]$ of 47 Tuc was varied by $\pm 0.1 \mathrm{dex}$.

The results are displayed in Table 1, where we give the percentage variation of each line index as a function of model input changes. To aid the reader in judging the importance of these effects, we provide in the last row of Table 1 the percentage variations due to an increment of $1 \mathrm{Gyr}$ in age (from 10 to 11 Gyrs). The best way to interpret the numbers in Table 1 is to compare the percentage variation in each index with the variation due to the age change given in the last row. For instance, $H \delta_{F}$ varies by $\pm 9 \%$ for an uncertainty of $\pm 50 \mathrm{~K}$ in the $T_{\text {eff }} \mathrm{s}$ of dwarfs. It varies by $-7 \%$ when age changes from 10 to 11 Gyrs. Therefore, the $\pm 50 \mathrm{~K}$ uncertainty in the $T_{\text {eff }} \mathrm{S}$ of dwarfs implies a $\mp 1.3 \mathrm{Gyr}$ uncertainty in the age inferred from $H \delta_{F}$ at the metallicity of 47 Tuc.

Before discussing systematic effects in the input stellar parameters, we assess the effect of the mismatch of the theoretical isochrones to the red giant branch of 47 Tuc in Figures 1 
and 2. In Section 2.1 we saw that the Salaris isochrone is bluer by $\sim 0.04$ mag at the lower giant branch and redder by a comparable amount at the upper giant branch. In the case of the Padova isochrones, the theoretical prediction is bluer than the observations through all the RGB, by $\sim 0.03$ mag. Such color differences, if due solely to temperature effects, translate into $T_{\text {eff }}$ mismatches of $\pm 50 \mathrm{~K}$ in the case of the Salaris isochrones and $+70 \mathrm{~K}$ in the case of the Padova isochrones, according to the calibration derived in Paper I. In the case of the Salaris isochrones, correcting for these mismatches amounts to negligible changes in the line index predictions. In fact, the percentage variations in all indices correspond to less than $0.4 \mathrm{Gyr}$, so that we do not need to perform any correction to our model predictions due to the slight mismatch in the red giant branch. This is because there is a compensation between the opposite $T_{\text {eff }}$ mismatches in the lower and upper giant branch. In the case of the Padova isochrones, as the model RGB is systematically bluer than the observations, the effect on spectroscopic age predictions is not negligible, leading to systematic age overestimates of about 1 Gyr. In section 3.4 we inferred a spectroscopic age of 14 Gyrs from $H \beta$ and slightly above that value from $H \gamma$. Correcting these predictions for the systematic temperature error in the RGB would bring them into better agreement with the age inferred from the position of the turn-off found in Section $2.1(12.5<\mathrm{t}<14.1$ Gyrs $)$.

Uncertainties due to systematic errors in the input parameters of dwarf stars are smaller than the variations of the input parameters of giants. Thus, they have only a relatively minor impact on model predictions, at the old ages of GCs. Except for $H \delta_{F}$, all line indices vary by less than $3 \%$ when varying the $T_{\text {eff }}$ and $[\mathrm{Fe} / \mathrm{H}]$-scales of dwarfs. Taking $H \beta$ as illustrative, this translates to roughly a $0.5 \mathrm{Gyr}$ uncertainty in age due to the $T_{e f f}$-scale of dwarfs and a $0.25 \mathrm{Gyr}$ uncertainty due to their $[\mathrm{Fe} / \mathrm{H}]$-scale. By comparison, uncertainties of $75 \mathrm{~K}$ or 0.1 dex in the input $T_{e f f}$ and $[\mathrm{Fe} / \mathrm{H}]$-scales of giants produce a $1 \mathrm{Gyr}$ uncertainty in the age inferred from either $H \beta$ or $H \delta_{F}$. This is particularly important in view of the current discrepancies in the $T_{\text {eff }}$-scales of giant stars among different sets of the most recent theoretical isochrones, which amount to $200 \mathrm{~K}$ (Salaris et al. 2002, Schiavon, Barbuy \& Bruzual 2000). Such a large discrepancy is still lingering, mostly because the position of the theoretical red giant branch is dependent on poorly constrained inputs of stellar evolutionary models, such as the mixing length parameter and the $\mathrm{T}(\tau)$ relation adopted as boundary condition for the interior models. On the other hand, the present work may be viewed as an important consistency check on the current theoretical Salaris $T_{\text {eff }} \mathrm{s}$, as Table 1 shows that an error of $\pm 200 \mathrm{~K}$ in the $T_{e f f} \mathrm{~S}$ of the giants would cause a mismatch of $\mp 3 \mathrm{Gyr}$ between the CMD and the spectroscopic ages, which is not seen. This is a valuable independent confirmation of the correctness of the Salaris $T_{e f f}$-scale, at least for giants.

The input metallicity of 47 Tuc is also an important source of uncertainty for spectroscopic age determination. Varying $[\mathrm{Fe} / \mathrm{H}]_{47 \mathrm{Tuc}}$ by \pm 0.1 dex (the nominal errorbar quoted 
by Carretta \& Gratton 1997) causes the spectroscopic age to vary by roughly \pm 1 Gyr, as measured from all Balmer lines. The effect of blue stragglers is also not negligible. If the observations are not corrected for the contribution of blue stragglers, ages are underestimated by slightly more than 1 Gyr when measured from $H \delta_{F}$. The effect on $H \gamma_{\sigma<130}$ and $H \beta$ is less important, leading to underestimates of $\sim 0.6$ and 0.5 Gyrs respectively.

\section{Conclusions}

In this paper, we compared model single stellar population spectra to the integrated spectrum of the metal-rich Galactic globular cluster 47 Tuc. The model spectra were computed from two sets of state-of-the-art theoretical isochrones. We tested the sensitivity of our predictions to variations in a number of key model inputs related to the spectral library employed, the theoretical isochrones, and the relations between fundamental and observed stellar parameters. Our main conclusions can be summarized as follows:

- Different sets of isochrones predict slightly different ages when compared to the cluster data on the color-magnitude diagram. In the case of the Salaris set, the isochrone providing the best match to the turn-off of the cluster has an age of 11-12 Gyrs. Vazdekis et al. (2001), using the same set of isochrones, obtained an age younger by about 1 Gyr. The difference is minor and is due to the different reddenings assumed. When the isochrones of Salasnich et al. (2000) are used, a slightly older age is obtained (12.5-14 Gyrs) the reason being that the latter set of isochrones does not consider diffusion of heavy elements. A final decision as to which set of isochrones is best awaits a better knowledge of the extent to which atomic diffusion is operating in main sequence stars.

- When compared to the observations in the luminosity function domain, both sets of theoretical isochrones adopted in this work underestimate the number of giant stars brighter than the horizontal branch. In the case of the Salaris isochrones, which do not include AGB stars, the mismatch amounts to 0.3-0.4 dex. The Padova set, even though it does include AGB stars, is also offset, though by a smaller amount $(\sim 0.2-0.3 \mathrm{dex})$. It is unclear to us what causes this discrepancy. At the level of the AGB bump, roughly $2 / 3$ of the mismatch seems to be due to the lack of AGB stars in the isochrones, this fraction being probably lower in other parts of the upper giant branch. However, even after performing an approximate correction

for the absence of AGB stars, there appears to be a small residual discrepancy between models and observations for first-ascent giants. We conclude that theoretical predictions to the luminosity functions of both the RGB and the AGB may be too low, though it is difficult to quantify by how much in each case. 
- Spectroscopic ages inferred from $H \beta$ and $H \gamma$ when adopting isochrones without any AGB stars are systematically older, by at least 3 Gyrs, than the ages inferred from the fit to the cluster's CMD. Moreover, metal-lines are systematically too weak and the integrated $(B-V)$ is too blue by 0.07 mag.

- Empirically correcting the theoretical LFs to match the observed luminosity function reduces the spectroscopic age to 11-12 Gyrs in the case of the Salaris isochrones, and $\sim 13$ Gyrs in the case of the Padova ones as determined from $H \beta$ and $H \gamma$ (the latter is obtained after correction for a systematic mismatch of the cluster's RGB by Padova isochrones). Such revised ages are now in excellent agreement with the ages inferred from fitting both sets of isochrones to the CMD. Also $(B-V)$ and metal-lines studied are very well fit by the LF-corrected models.

- The best value found from the fit to $H \delta_{F}$ is still higher by $\sim 3$ Gyrs than found for the other Balmer lines. This mismatch is in the same sense found in Paper I, where $H \delta_{F}$ computed directly from the CMD-based synthesis was relatively stronger than $H \gamma$ or $H \beta$. As $H \delta_{F}$ is more strongly affected by $\mathrm{C}, \mathrm{N}$ abundance anomalies, we suggested in Paper I that the discrepancy was due to our failure to properly model and correct for $\mathrm{CN}$ lines within the passband and continuum windows of the index.

- We estimate the impact on spectroscopic ages of uncertainties in a number of model inputs, such as the $T_{\text {eff }}$ and $[\mathrm{Fe} / \mathrm{H}]$-scales of dwarfs and giants, the luminosity function of the red giant branch and the metallicity of the cluster. Spectroscopic age-dating of old stellar populations is mostly influenced by uncertainties coming from the $T_{\text {eff }}$ and $[\mathrm{Fe} / \mathrm{H}]$ scale of giant stars, as well as the luminosity function of the upper red giant branch and the metallicity adopted for the cluster. Using $H \beta$, a 1 Gyr uncertainty is caused by an error of $75 \mathrm{~K}$ in the giant $T_{\text {eff }}$-scale, a 0.1 dex error in the giant-branch luminosity function, and a 0.1 dex error in either the $[\mathrm{Fe} / \mathrm{H}]$-scale of giants or the $[\mathrm{Fe} / \mathrm{H}]$ of the stellar population. Because the uncertainties in the parameters of dwarf stars are lower, their impact on model predictions is generally less important.

- The contribution by blue stragglers to the integrated line indices is important only for $H \delta_{F}$ and slightly less so for $H \gamma_{\sigma<130}$. When $H \delta_{F}$ is corrected for the contribution of blue stragglers, we obtain an age $1 \mathrm{Gyr}$ older. In the case of $H \gamma_{\sigma<130}$, the age inferred is $0.5 \mathrm{Gyr}$ older.

In summary, we conclude that the exceedingly high spectroscopic ages found for 47 Tuc in previous works was due to: 1) the use of theoretical isochrones that neglect important effects such as He-diffusion and $\alpha$-enhancement, as shown by Vazdekis et al. (2001), and 2) the underestimate of the number of giant stars above the horizontal branch, which was 
partly due to the omission of AGB stars from the isochrones, and partly to a genuine apparent shortfall in the predicted numbers of AGB stars and, most likely, first-ascent giants as well. Another requirement, which is discussed in Paper I, is on the iron abundance of the cluster. Models provide a good match if $[\mathrm{Fe} / \mathrm{H}]$ is 0.05 dex lower than found by Carretta \& Gratton (1997). Such a shift in $[\mathrm{Fe} / \mathrm{H}]$ is well within the errorbars of Carretta \& Gratton's analysis and the uncertainties in the $[\mathrm{Fe} / \mathrm{H}]$-scale of our stellar library.

With regard to our long term goal of constructing metal-rich stellar population models for elliptical galaxies, we have made considerable progress. First, the ages from $H \beta$ and $H \gamma_{\sigma<130}$ agree, after the latter is corrected for the effect of $\mathrm{C}, \mathrm{N}$ abundance variations (see Paper I). Second, all metal lines are correctly predicted for the same ages obtained from Balmer lines. Third, the giant temperature scale of the Salaris models looks to be generally correct. On the other hand, $H \delta_{F}$ is shown to be a problematic feature, embedded as it is in a forest of CN lines. This problem is likely to be further exacerbated in elliptical galaxies, which have notoriously strong $\mathrm{CN}$ features. Moreover, the present models do not include variations in Type Ia vs. Type II supernovae element ratios (Trager et al. 1998), nor do they probe the high-metallicity regime needed for ellipticals. Finally, we have identified an error in the upper giant branch luminosity function of current evolutionary models, which underestimate the aggregate number of luminous red giants in 47 Tuc by roughly a factor of 2. We have furthermore shown that an accurate LF for these stars is needed for accurate spectroscopic age determination. A major unknown is whether the red-giant excess of 47 Tuc extends to all metal-rich old populations, and what its magnitude might be. Testing the luminosity function in old metal-rich populations has emerged as an important prerequisite for stellar population studies. These and further improvements are left for future works.

We would like to thank Maurizio Salaris for making his isochrones available. Stefano Covino is thanked for the red integrated spectrum of 47 Tuc. We would also like to thank Alexandre Vazdekis, Maurizio Salaris, Manuela Zoccali, Achim Weiss and Mike Bolte for helpful discussions. The referee, Brad Gibson, is thanked for valuable suggestions that greatly improved this paper. R.P.S. thanks the hospitality of the Physics Dept. of the University of North Carolina, Chapel Hill, where part of this work was developed. Likewise, J.A.R. thanks the Astronomy Department at UC, Santa Cruz for hospitality during a visit in which part of this work was developed. This work has made extensive use of the Simbad database. R.P.S. acknowledges support provided by the National Science Foundation through grant GF-1002-99 and from the Association of Universities for Research in Astronomy, Inc., under NSF cooperative agreement AST 96-13615, and CNPq/Brazil, for financial help (200510/991). This research has also been supported by NSF grant AST-9900720 to the University of North Carolina, and by NSF grants AST-9529098 and AST-0071198 to the University of 
California, Santa Cruz.

\section{REFERENCES}

Albrow, M.D., Gilliland, R.L., Brown, T.M., Edmonds, P.D., Guhathakurta, P. \& Sarajedini, A. 2001, ApJ, 559, 1060

Alonso, A., Arribas, S. \& Martínez-Roger 1995, A\&A, 297, 197

Alonso, A., Arribas, S. \& Martínez-Roger 1996, A\&A, 313, 873

Alonso, A., Arribas, S. \& Martínez-Roger 1999, A\&AS, 139, 335

Basu, S., Pinsonneault, M.H. \& Bahcall, J.N. 2000, ApJ, 529, 1084

Bergbusch, P.A. \& Vandenberg, D.A. 1992, ApJS, 81, 163

Bolte, M. 1994, ApJ, 431, 223

Carretta, E. \& Gratton, R.G. 1997, A\&AS, 121, 95

Cassisi, S., Castellani, V., Degl'Innocenti, S., Piotto, G \& Salaris, M. 2001, A\&A, 366, 578

Chaboyer, B., Fenton, W.H., Nelan, J.E., Patnaude, D.J. \& Simon, F.E. 2001, ApJ, 562, 521

Cohen, J.G., Blakeslee, J.P. \& Ryzhov, A. 1998, ApJ, 496, 808

Deliyannis, C.P. \& Demarque, P. 1991, ApJ, 379, 216.

Gibson, B.K., Madgwick, D.S., Jones, L.A., Da Costa, G.S. \& Norris, J.E. 1999 AJ, 118, 1268

Gratton, R.G. et al. 2001, A\&A, 369, 87

Hesser, J.E., Harris, W.E., Vandenberg, D.A., Allwright, J.W.B., Shott, P. \& Stetson, P.B. 1987, PASP, 99, 739

Howell, J.H., Guhathakurta, P. \& Gilliland, R.L. 2000, PASP, 112, 1200

Jones, L.A. 1999, PhD Thesis, University of North Carolina

Kaluzny, J., Wyzocka, A., Stanek, K.Z. \& Krzemiński, W. 1998, AcA, 48, 439 
Langer, G.E., Bolte, M. \& Sandquist, E. 2000, ApJ, 529, 936

Lebreton, Y., Perrin, M.-N., Cayrel, R., Baglin, A. \& Fernandes, F. 1999, A\&A, 350, 587

Maraston, C. 1998, MNRAS, 300, 872

Richer, H.B., et al. 1996, ApJ, 463, 602

Rose, J.A. 1994, 107, 206

Rubenstein, E.P. \& Bailyn, C.D. 1999 ApJ, 513, L33

Salaris, M. \& Weiss, A. 1998 A\&A, 335, 943

Salaris, M. \& Weiss, A. 2001 A\&A, 376, 955

Salaris, M., Cassisi, S. \& Weiss, A. 2002, PASP, 114, 375

Salasnich, B., Girardi, L., Weiss, A. \& Chiosi, C. 2000, A\&A, 361, 1023

Sandquist, E., Bolte, M., Stetson, P.B. \& Hesser, J.E. 1996, ApJ, 470, 910

Sandquist, E., Bolte, M., Langer, G.E., Hesser, J.E. \& Mendes de Oliveira, C. 1999, ApJ, 518,262

Schiavon, R.P., Barbuy, B. \& Bruzual A., G. 2000 ApJ, 532, 453

Schiavon, R.P., Faber, S.M., Rose, J.A. \& Castilho, B.V. 2002, ApJ, submitted (Paper I)

Soubiran, C., Katz, D. \& Cayrel, R. 1998, A\&AS, 133, 221

Spite, F. \& Spite, M. 1982, A\&A, 115, 357

Stetson, P.B. 1991, in The Formation and Evolution of Star Clusters, ed. K. Janes (ASP Conf. Ser. 13), 88

Straniero, O., Chieffi, A. \& Limongi, M. 1997, ApJ, 490, 425

Trager, S.C., Worthey, G., Faber, S.M. Burstein, D. \& González, J.J. 1998, ApJS, 116, 1

Vazdekis, A., Salaris, M., Arimoto, N. \& Rose, J.A., 2001, ApJ, 549, 274

Weiss, A. \& Salaris, M. 1999 A\&A, 346, 897

Westera, P., Lejeune, T., Buser, R., Cuisinier, F. \& Bruzual, G. 2002, A\&A, 381, 524

Zoccali, M. \& Piotto, G. 2000, A\&A, 358, 943 
Zoccali, M., Cassisi, S., Bono, G., Piotto, G., Rich, R.M. \& Djorgovski, S.G. 2000, ApJ, 538, 289 


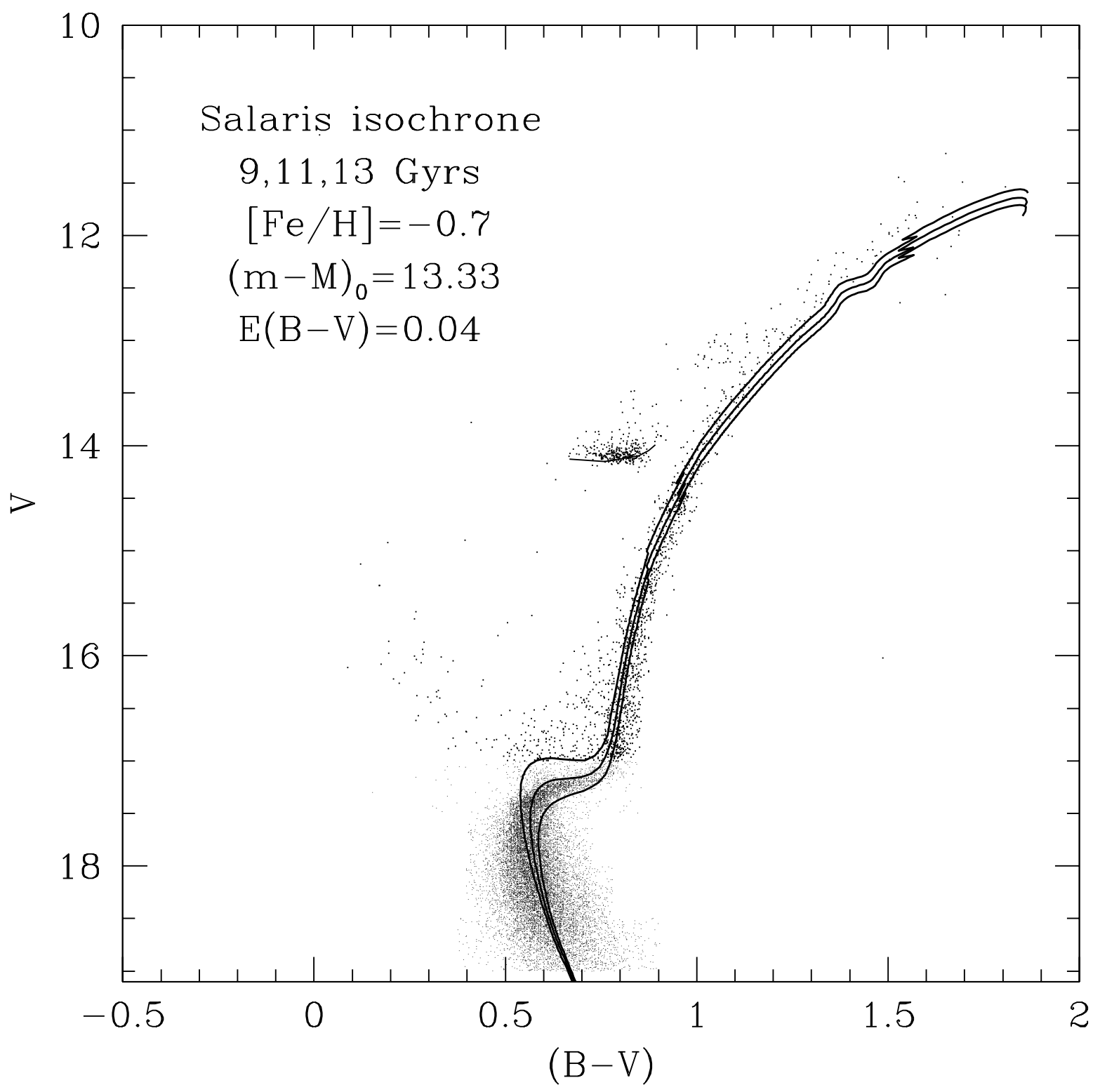

Fig. 1.- CMD of 47 Tuc out to a radius of $\sim 30$ " from Howell et al. (2000). The theoretical isochrones are described in Vazdekis et al. (2001). They have been converted to the observational plane by adopting the calibrations of Alonso et al. $(1995,1996)$ for dwarfs and the calibration presented in Paper I for the giants. Adopted values for the distance modulus and $E(B-V)$ are displayed in the upper left corner and were taken from Kaluzny et al. (1998). The best-fitting age according to those isochrones is 11-12 Gyrs. 


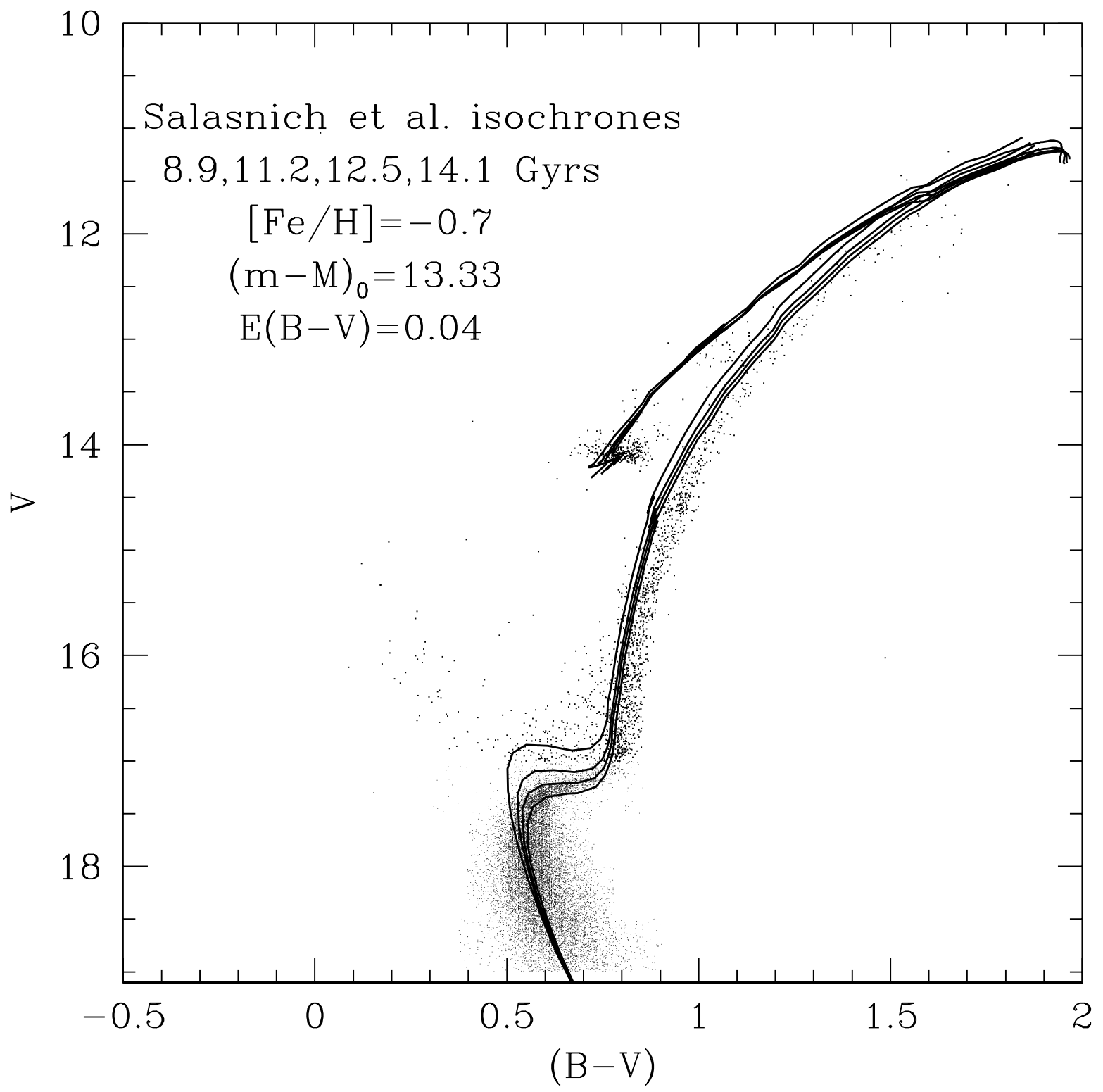

Fig. 2.- Same as Figure 1, but now comparing the data on 47 Tuc with the isochrones from Salasnich et al. (2000). Those isochrones were computed using the same chemical composition of the Salaris isochrones but do not include diffusion of heavy elements. For that reason, the age that best fits the turn-off color and luminosity is $\sim 1$ Gyr older than in the case of the Salaris isochrone. 


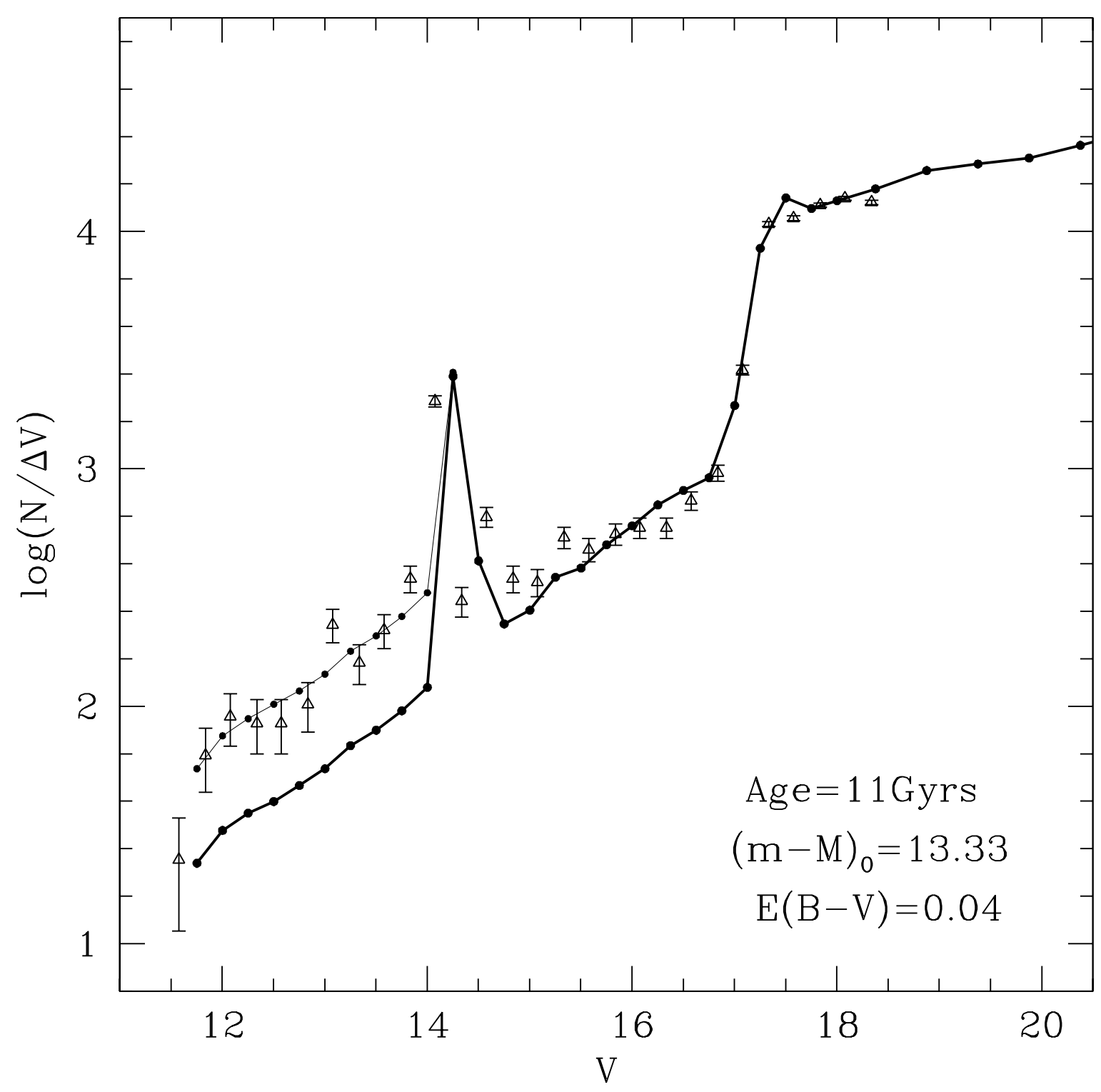

Fig. 3.- Comparison between the observed luminosity function of 47 Tuc and that obtained from the best fitting Salaris isochrone. The thick line represents the original Salaris LF, which lacks AGB stars. The thin line represents our "corrected" LF, in which a constant factor of 0.4 dex has been applied to the number counts brighter than $\mathrm{V} \sim 14$ in order to bring them into accordance with the observed LF. The secondary peak in the observational data at $\mathrm{V}$ $\sim 13$ is the AGB bump. 


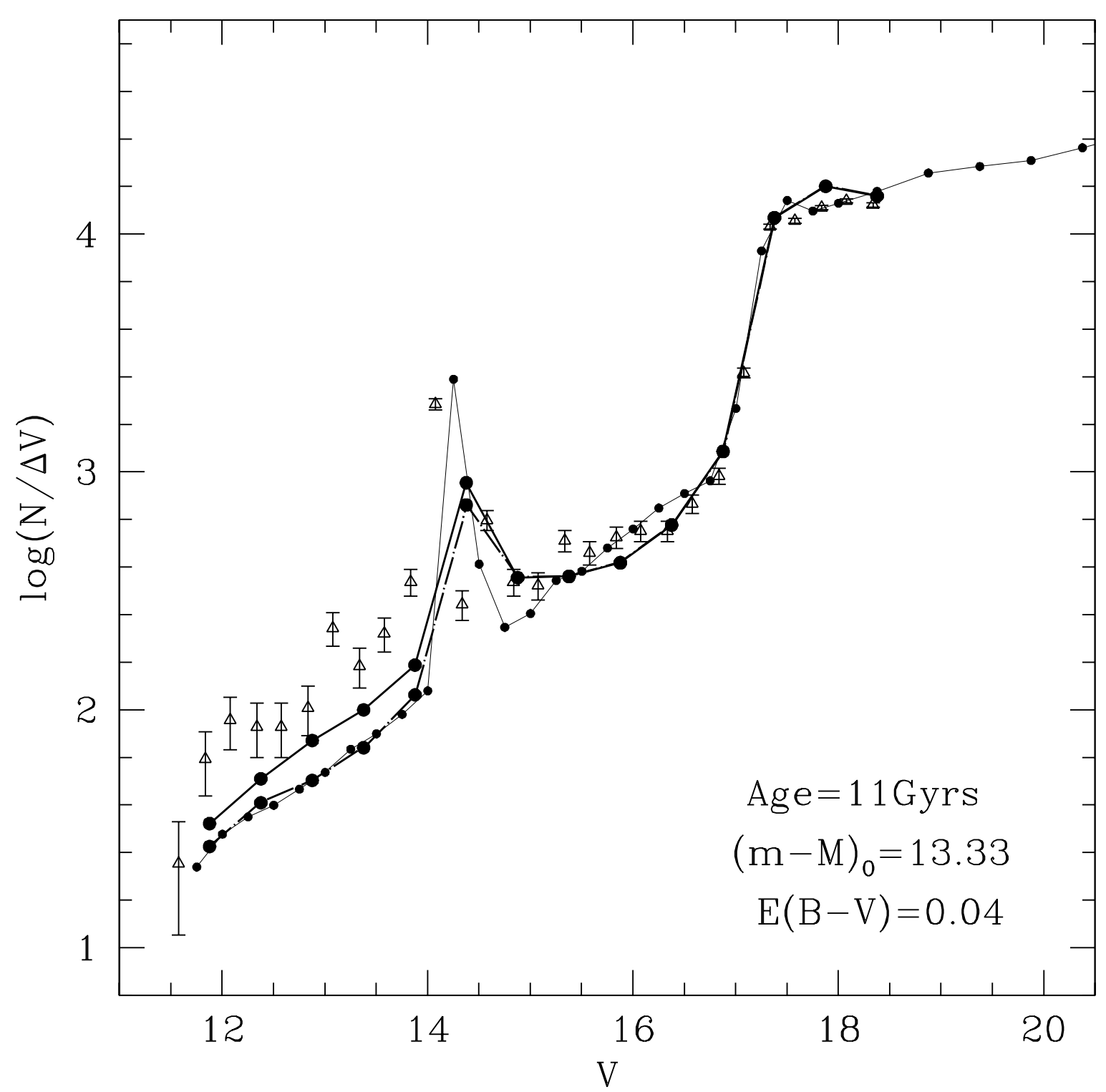

Fig. 4.- The observed LF of 47 Tuc versus the LF from the Padova isochrones (Salasnich et al. 2000). The thick solid line represents the original LF, while the thick dash-dotted line represents the LF that results when AGB stars are artificially removed. For reference, we repeat from Figure 3 the LF inferred from the Salaris isochrone (thin line). The broader Horizontal Branch in the Padova isochrones $(\mathrm{V} \sim 14.5)$ is due to their coarser mass bins, but total number counts in the Horizontal Branch are the same. When stripped of AGB stars, the Padova LF is in very good agreement with the one from Salaris. However, the full Padova isochrone, which does include AGB stars, still underpredicts the observed number counts above the Horizontal Branch by approximately 0.3 dex. 


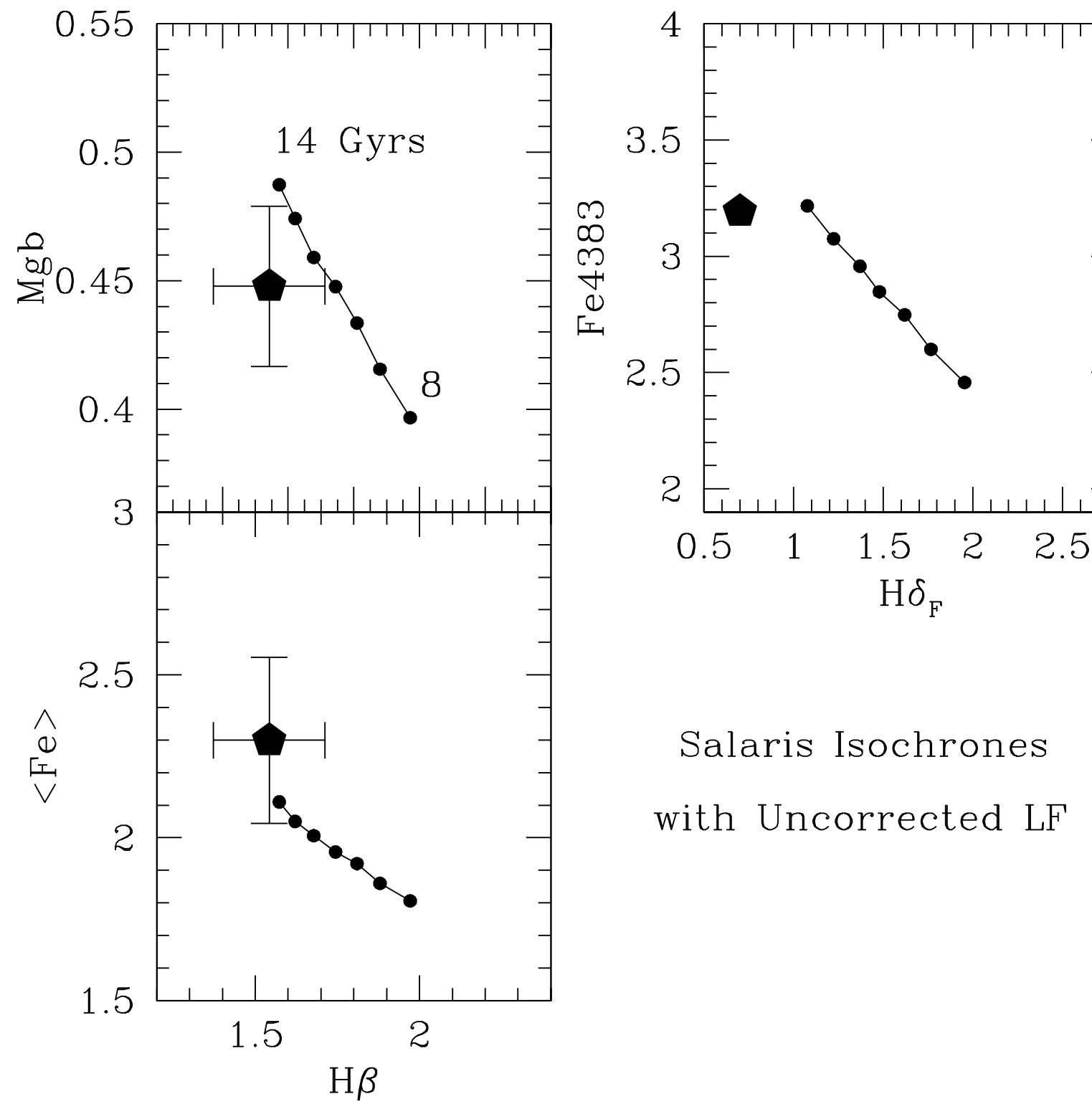

Fig. 5.- Comparison between model predictions and observed absorption line indices, corrected to eliminate the contribution by blue stragglers. Small filled circles connected by solid lines represent the computations based on the original Salaris isochrones, no AGB included, (see description in text) for ages varying, from left to right, between 14 and 8 Gyr. The isochrones have originally been computed for $[\mathrm{Fe} / \mathrm{H}]=-0.7$, but model predictions are corrected, according to Paper I, to $[\mathrm{Fe} / \mathrm{H}]=-0.75$. Other corrections to model predictions are also applied, namely, for the paucity of metal-poor library giants and the effect of CN-strong stars, also as described in Paper I. The best-fitting spectroscopic age according to $H \beta$ is 14 Gyr (2-3 Gyrs older than the best CMD-fitting age). For $\mathrm{H} \delta_{F}$, the best fitting age is well in excess of 14 Gyr. Finally, the observed indices have had the contribution by blue stragglers subtracted, as indicated in Table 4 of Paper I. 


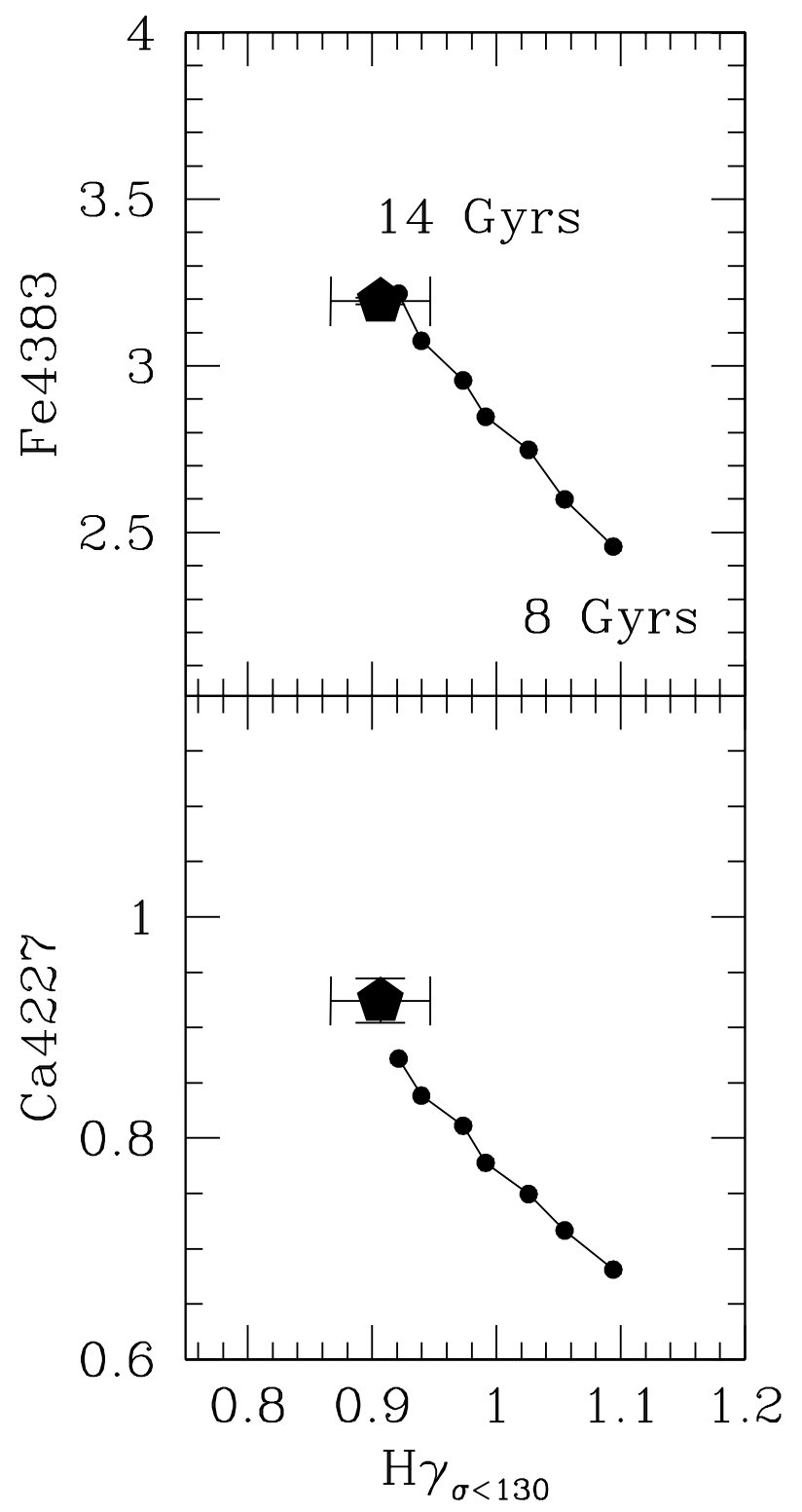

\section{Salaris Isochrones with Uncorrected LFs}

Fig. 6.- Same as Figure 5 for other combinations of line indices. The models indicate an age of 14 Gyr based on $H \gamma$. 


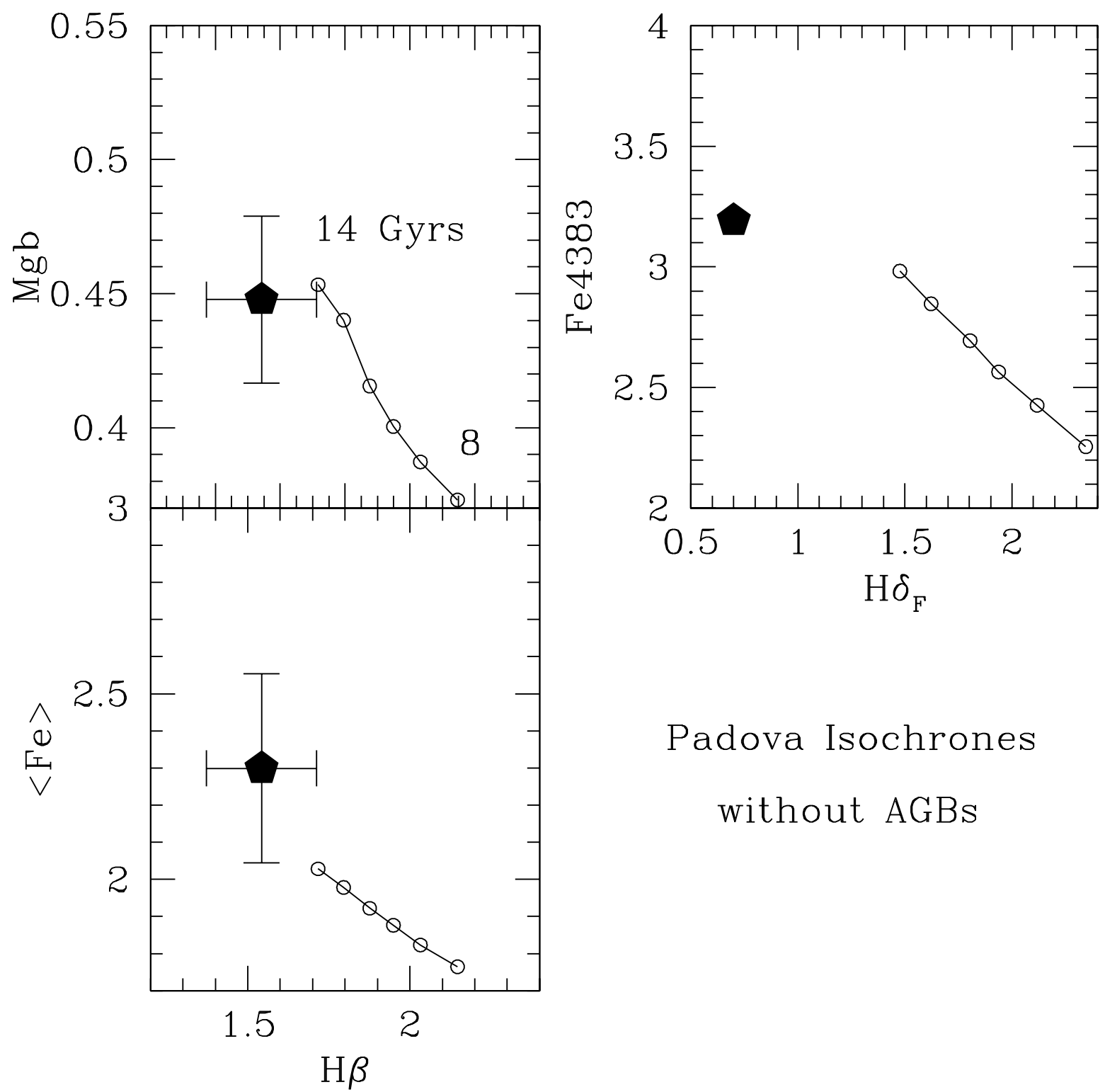

Fig. 7.- Same as Figure 5, now showing model predictions from the Salasnich et al. (2000) isochrones, excluding the contribution of AGB stars. As expected, the ages inferred are older than those based on Salaris isochrones by $\sim 2-3$ Gyrs. This is partly because Padova isochrones do not consider diffusion of heavy elements, and partly because they have slightly warmer giant branches (see text). 


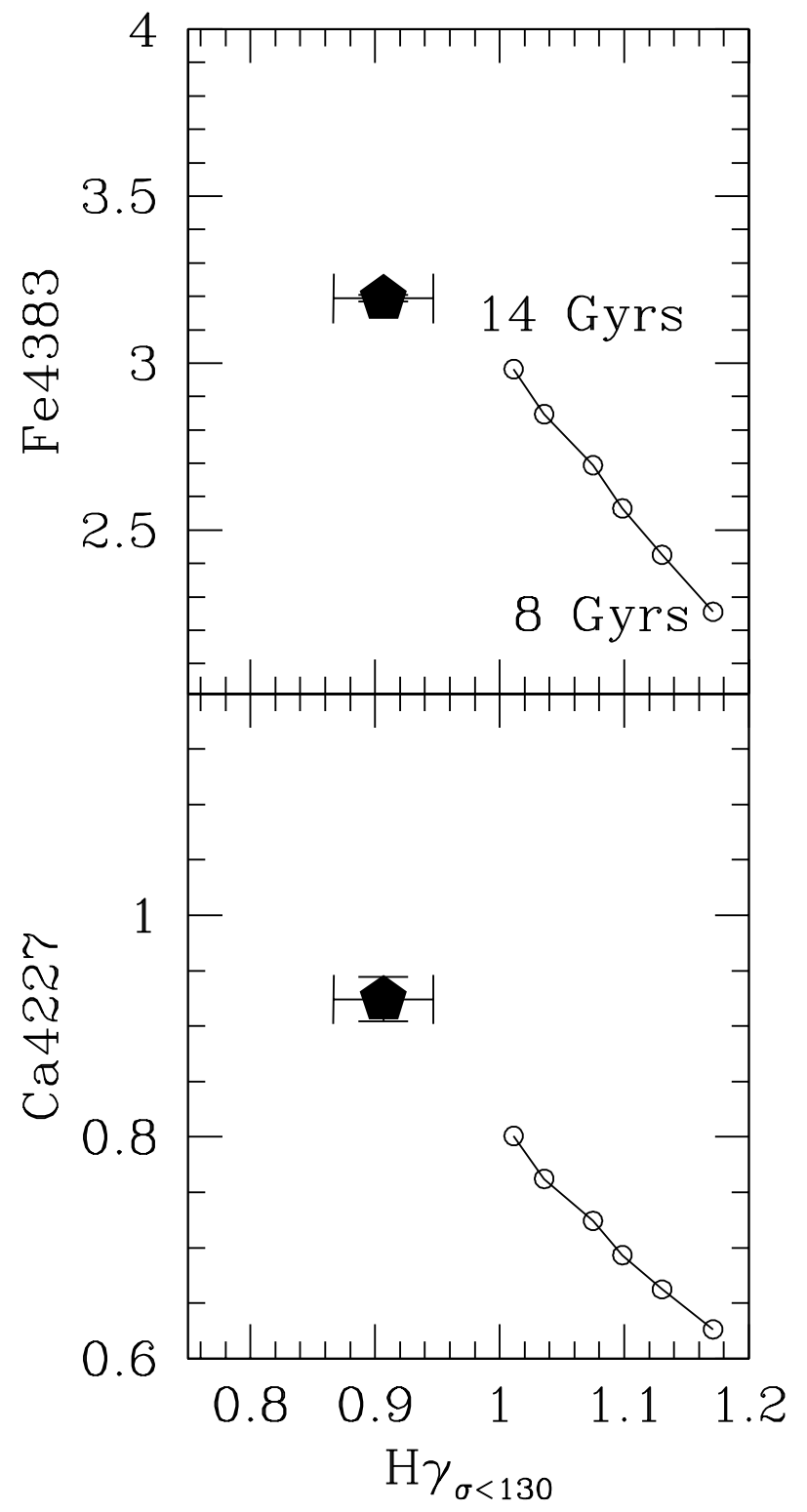

\section{Padova Isochrones} without AGBs

Fig. 8. - Same as Figure 7 for other combinations of line indices. 


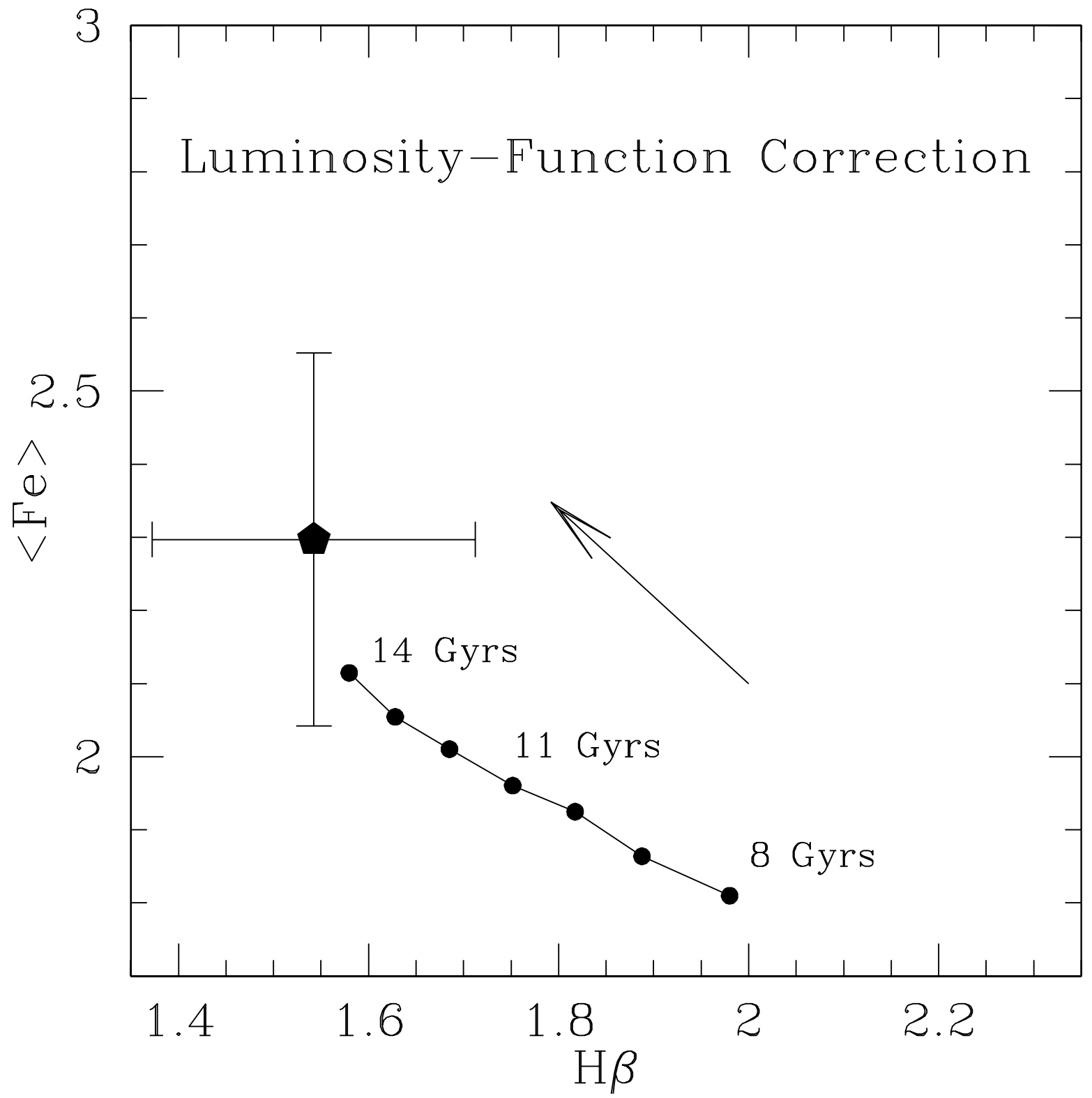

Fig. 9.- The $<F e>$ vs. $H \beta$ panel repeated from 5. The solid dots are the model predictions using the Salaris isochrones with their original, uncorrected LFs. The arrow indicates the extent and direction by which the model predictions change if the theoretical giant-branch LF is corrected according to the thin line in Figure 3. In general, because of the increase of red-giant light contribution, metal lines become stronger and hydrogen lines become weaker. The new age estimated from $H \beta$ is now only 11-12 Gyrs, bringing the spectroscopic age into good agreement with the isochrone age from Figure 1. 

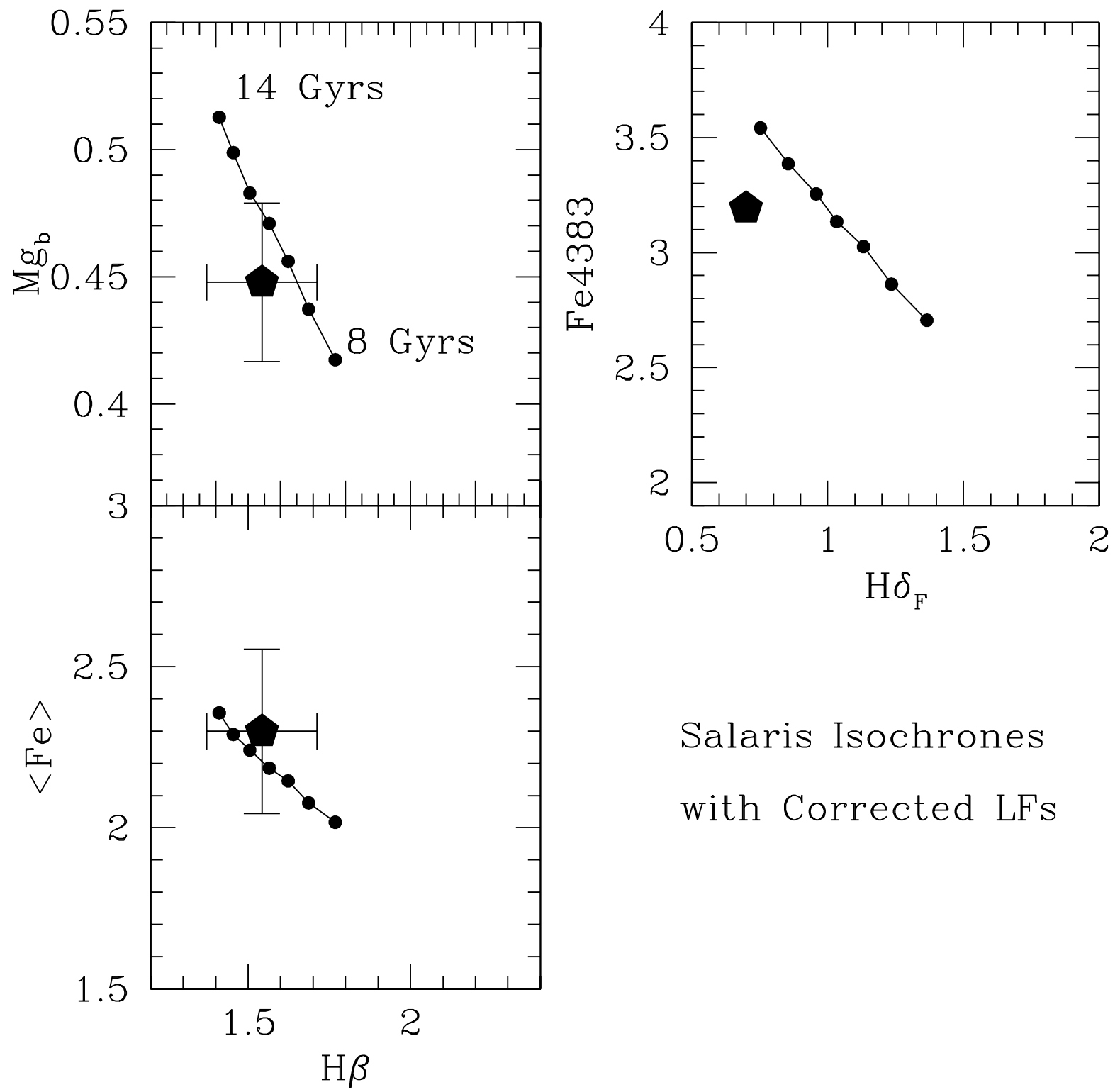

Salaris Isochrones with Corrected LFs

Fig. 10.- Same as Figure 5 but now using corrected LFs. The models shown in Figure 5 have been shifted analogously to the arrow in Figure 9. The correction brings the spectroscopic age for $H \beta$ into agreement with CMD-based age $\sim 11$ Gyrs. $\mathrm{H} \delta_{F}$ still gives a slightly higher age ( $\sim 14$ Gyrs), as discussed in the text and in Paper I. 


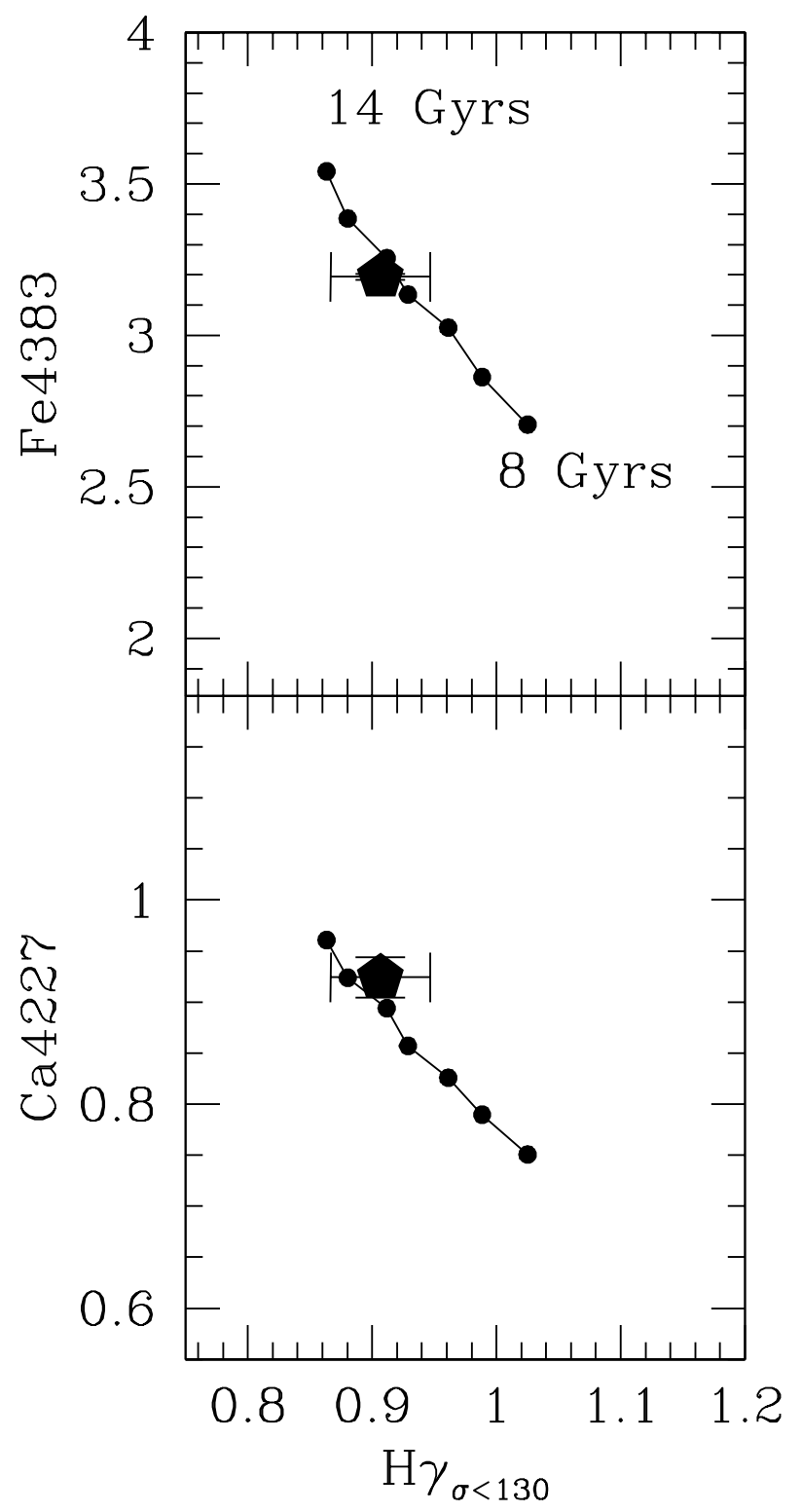

Salaris Isochrones with Corrected LFs

Fig. 11.- Same as Figure 10, for other combinations of line indices. For $H \gamma_{\sigma<130}$ the models predict an age in agreement with the CMD-based and $H \beta$-based ages, namely, 12 Gyrs. 

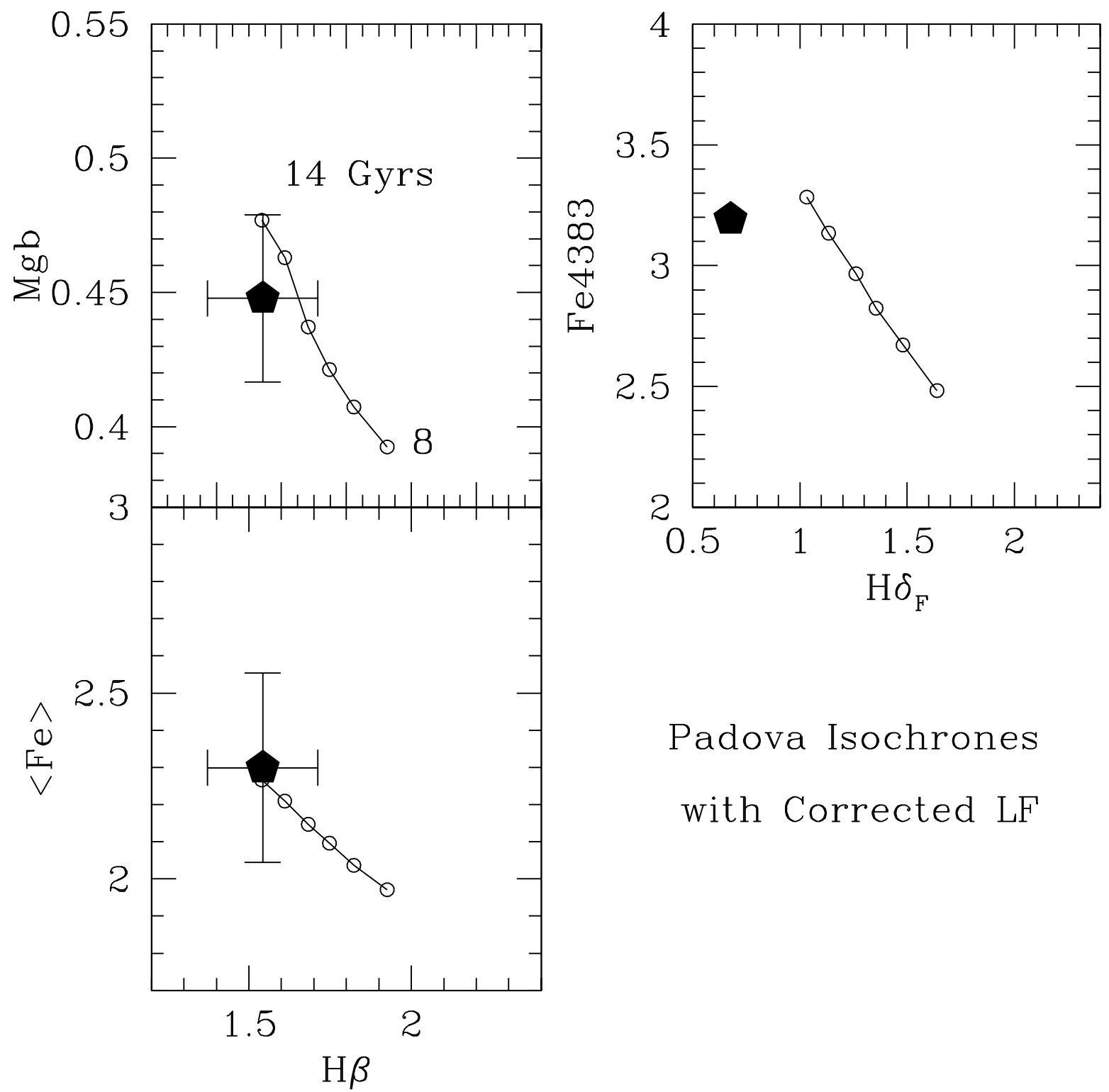

\section{Padova Isochrones with Corrected LF}

Fig. 12.- Same as Figure 7 but now using corrected LFs. The models shown in Figure 7 have been shifted analogously to the arrow in Figure 9. The correction reduces the spectroscopic age for $H \beta$ to $\sim 14$ Gyrs, in agreement with the CMD-based age for Padova isochrones (2). As in the case of Salaris isochrones, the spectroscopic age based on $\mathrm{H} \delta_{F}$ is higher. 


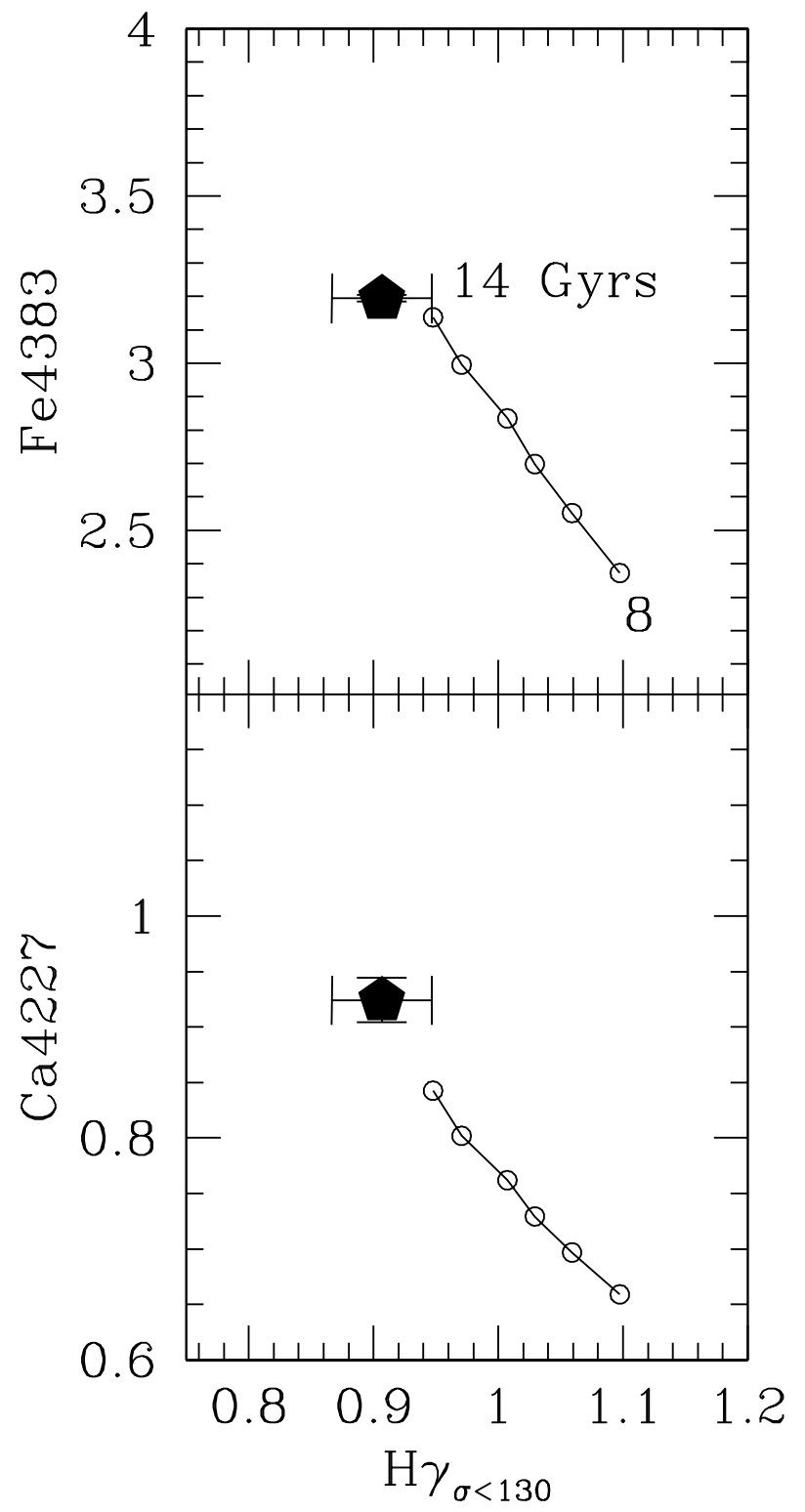

\section{Padova Isochrones with Corrected LFs}

Fig. 13.- Same as Figure 12, for other combinations of line indices. For $H \gamma_{\sigma<130}$ the LF-corrected Padova models predict a spectroscopic age which is consistent with the CMDbased and $H \beta$-based ages, namely, $\sim 14$ Gyrs. Because of the absence of heavy-element diffusion in the Padova isochrones, and because their giant branches are slightly warmer, the spectroscopic age inferred is older than the one based on Salaris isochrones by $\sim 2$ Gyrs. 
Table 1. Variation of line indices (\%) as a function of uncertainties in model input parameters. In the last row, percentage variations are given for a change from 10 to 11 Gyrs, according to our models.

\begin{tabular}{ccccccccc}
\hline \hline$H \delta_{F}$ & $\mathrm{Ca} 4227$ & $H \gamma_{\sigma<130}$ & $\mathrm{Fe} 4383$ & $H \beta$ & $M g_{b}$ & $M g_{2}$ & $\langle F e>$ & \\
\hline \pm 4 & $\mp 11$ & \pm 1.5 & $\mp 4$ & \pm 4.5 & $\mp 6$ & $\mp 8.5$ & $\mp 3.5$ & $T_{\text {Giants }} \pm 75 \mathrm{~K}$ \\
\pm 9 & $\mp 1$ & \pm 1.5 & $\mp 1.5$ & \pm 1.5 & $\mp 1$ & $\mp 1$ & $\mp<1$ & $T_{\text {Dwarfs }} \pm 50 \mathrm{~K}$ \\
$\mp 11$ & \pm 2 & \pm 2 & \pm 5 & \pm 3 & \pm 3.5 & \pm 5.5 & \pm 4.5 & {$[\mathrm{Fe} / \mathrm{H}]_{\text {Giants }} \pm 0.1$} \\
$\mp 2.5$ & \pm 1 & \pm 1 & \pm 1.5 & $\pm<1$ & \pm 1 & \pm 1 & \pm 1 & {$[\mathrm{Fe} / \mathrm{H}]_{\text {Dwarfs }} \pm 0.05$} \\
$\mp 7.5$ & \pm 2.5 & \pm 4 & \pm 6 & \pm 5 & \pm 4 & \pm 5 & \pm 5 & {$[\mathrm{Fe} / \mathrm{H}]_{47 T u c} \pm 0.1$} \\
-30 & +10 & -6 & +10 & -10 & +5 & +12 & +11.5 & LF Correction \\
-9.5 & +1 & -2 & +1 & -2 & $<1$ & $<1$ & $<1$ & No BS \\
-7 & +4 & -3 & +4 & -4 & +4 & +2 & +2 & $10 \rightarrow 11$ Gyrs \\
\hline
\end{tabular}

